\title{
Rudolf Ladenburg and the first quantum interpretation of optical dispersion
}

\author{
Marta Jordi Taltavull ${ }^{\mathrm{a}}$ \\ Institute of Mathematics, Johannes Gutenberg University Mainz, 55128 Mainz, \\ Germany
}

Received 3 June 2020 / Accepted 27 July 2020

Published online 28 September 2020

(C) The Author(s) 2020. This article is published with open access at Springerlink.com

\begin{abstract}
In 1921, the experimental physicist Rudolf Ladenburg put forward the first quantum interpretation of optical dispersion. Theoretical physicists had tried to explain dispersion from the point of view of quantum theory ever since 1913, when Niels Bohr proposed his quantum model of atom. Yet, their theories proved unsuccessful. It was Ladenburg who gave a breakthrough step toward our quantum understanding of dispersion. In order to understand Ladenburg's step, I analyze Ladenburg's experimental work on dispersion prior to 1913, the reasons why the first theories of dispersion after 1913 were not satisfactory, and Ladenburg's 1921 proposal. I argue that Ladenburg's early experimental work on dispersion is indispensable to understand his 1921 paper. The specific kind of experiments he performed before 1913, the related interpretative problems, and the way he tried to solve them, led him reapproach the dispersion problem in 1921 in a way that was completely different from the way theoretical physicists had done it before.
\end{abstract}

\section{Introduction}

In 1921, the German experimentalist Rudolf Ladenburg put forward the first quantum interpretation of optical dispersion, in terms of Bohr's 1913 quantum atomic model. This was a great achievement, for optical dispersion had been ever since 1913 one of the key optical phenomena that resisted any quantum explanation. Prominent theoretical physicists like Peter Debye and Arnold Sommerfeld had developed in 1915 new theories of dispersion that retained the advantages of the classical theories while being adapted to Bohr's atomic model. Yet these theories collapsed soon afterwards.

According to secondary sources, Ladenburg's 1921 quantum reinterpretation played a crucial role in the first quantum theory of dispersion laid down in 1924 by Hendrik Kramers, and more generally to the foundations of matrix mechanics established in 1925 by Werner Heisenberg. The relationship between Ladenburg's 1921 and

a e-mail: majordit@uni-mainz.de; martajordit@gmail.com 
Heisenberg's matrix mechanics has been already historically explored. ${ }^{1}$ Yet it remains to be analyzed how Ladenburg conceived his original idea for the 1921 paper. Moreover, Bohr, Kramers, and Heisenberg were theoretical physicists, whereas Ladenburg was an experimentalist. How could an experimental physicist come up with such an important and influential idea for further theoretical developments in quantum physics?

The answer presented in this paper is that Ladenburg could tackle the problem in such a different way from theoreticians precisely because he was an experimentalist, also theoretically skilled, working on specific features of dispersion ever since 1908 . He was an original thinker who could do easy calculations, though not complicated mathematical developments. Two aspects of Ladenburg's research prior to 1914 are important to understand Ladenburg's 1921 approach to dispersion. First, the specific kind of experiments he performed combined optical dispersion with spectral lines. Spectral lines were later explained by Bohr's atomic model. Second, Ladenburg had not only performed experiments of optical dispersion with great precision, but he had also been deeply concerned with interpretative issues. Given classical theories of the phenomenon, dispersion occurred because light and matter resonated at certain frequencies. By assuming a certain kind of particles, it was possible to interpret the empirical parameters in terms of the number of these resonating particles. That is, optical phenomena allowed scientists to investigate the microscopic structure of matter. In trying to interpret his experimental results in this way it was that Ladenburg encountered the first interpretative difficulties, which did not undermine the general picture of resonance. The problem was the specific way in which resonance was supposed to take place within atoms and molecules, whether light interacted directly with individually vibrating particles within atoms and molecules or whether light interacted with atoms and molecules as a whole. Ladenburg did not stop there. In 1914, he enlarged his conceptual resources to find a better way to formulate the interpretative problem that paved the way for his 1921 reinterpretation.

After the Great War, upon becoming an advocate of Bohr's atomic theory, Ladenburg was in the best position to look at optical dispersion in a radically different way from theoreticians. Debye's and Sommerfeld's strategy had been to look for a new theory of dispersion that made resonance conceptually compatible with Bohr's atomic model. They did so by modeling light waves interacting with individually resonating electrons. Remarkably, Ladenburg's experiments of dispersion around the spectral lines were out of the validity range of Debye's and Sommerfeld's theoretical accounts. In contrast to Debye and Sommerfeld, Ladenburg did not look for a new theory that solved the deep conceptual issues between classical and quantum theories. What Ladenburg aimed at was solving the previous interpretative problem of optical dispersion. For that purpose, he used his arsenal of conceptual resources gathered previously to WWI and he simply gave the old parameters a new physical meaning in terms of Bohr's atomic model, without worrying about questions of conceptual consistency.

In order to understand Ladenburg's 1921 quantum reinterpretation of dispersion on the grounds of his previous work, I have divided the paper in four parts. In Section 2, I explain the commonplace theoretical understanding of dispersion at the turn of the 20th century and I describe the experimental problem of dispersion around spectral lines before Ladenburg took it up in 1908. In Section 3, I analyze Ladenburg's

\footnotetext{
${ }^{1}$ See above all (Duncan, 2007a), (Janssen, 2019), and chapters 6 and 7 of (Jordi Taltavull, 2019). Until 2017, the standard account was that the theory of dispersion was the most important step towards the development of matrix mechanics (MacKinnon, 1977; Dresden, 1987; Darrigol, 1992; Duncan, 2007a). In 2017, this view was challenged. According to (Blum, 2017), the immediate step towards Heisenberg's 1925 paper leading to matrix mechanics was not dispersion, but Heisenberg's unpublished work on multiplet intensities. Nevertheless, the new historical account does not undermine the quantum theory of dispersion as an important source for inspiration in previous stages of Heisenberg's thinking. It rather calls for a multicausal story.
} 
work on dispersion from 1908 to 1914, until he had to leave his research on optics due to WWI. In Section 4, I summarize Debye's and Sommerfeld's contributions to optical dispersion as a reflection on the reasons why dispersion was such a difficult phenomenon to interpret from the point of view of Bohr's atomic model. Eventually, in Section 5, I expound Ladenburg's 1921 idea and I relate it to his previous contributions.

\section{Optical dispersion before Rudolf Ladenburg}

\subsection{Optical dispersion and the origin of the resonance model in optics: 1870-1900}

When Ladenburg started to work on optical dispersion in 1908, this was a well-known phenomenon with a long history and well-established theoretical grounds.

Optical dispersion was the spread of light into different colors when passing through a prismatic medium. It was thought to be a consequence of the change of velocity of light depending on its color within media, different from that in vacuum. This change was quantified through the index of refraction. Thus, optical dispersion amounted to the variation of the index of refraction as a function of light frequency or wavelength. Until 1870, optical dispersion had always been represented by a continuous decrease of the index of refraction for increasing wavelength, which meant the following order of dispersed colors: red, orange, yellow, green, blue, and violet.

In the early 1870s, several observations undermined this expected behavior. More specifically, the Danish physicist Christian Christiansen and the German physicist August Kundt discovered that, through liquid dyes, dispersion was not continuous, but it presented discontinuities at specific positions of the spectrum. A different order of colors was observed in these cases (Christiansen, 1870; Christiansen, 1871; Kundt, 1871a). Liquid dyes had the particularity that they absorbed light at certain frequencies, the so-called "surface colors," and they were transparent for the rest of the spectrum. The point is that discontinuities in optical dispersion took place exactly at the surface colors (see Fig. 1 for the discontinuous behavior) (Kundt, 1871b; Kundt, 1871c; Kundt, 1872), namely, at the absorption frequencies. ${ }^{2}$

Explaining optical dispersion called for a new conceptual framework, in which optical properties of matter arose from the motion of microscopic particles of matter interacting with light. Before that, it was commonplace that light was the propagation of the mechanical vibrations of an elastic and transparent substance filling everything, the so-called ether. Matter was supposed to modify the propagation of this vibrations, yet not generate or absorb them. ${ }^{3}$ In the early 1870 s, this situation changed. In 1872 , the practically unknown German physicist Wilhelm Sellmeier proposed a new theory of optical dispersion that led to a paradigmatic change in the way light-matter interactions were conceived (Sellmeier, 1872a; Sellmeier, 1872b; Sellmeier, 1872c; Sellmeier, 1872d). ${ }^{4}$ For the first time, hypothetical microscopic matter particles were supposed to vibrate at specific frequencies and therefore actively participate in the generation

\footnotetext{
${ }^{2}$ For more information about this episode, see (Jordi Taltavull, 2016, 318-321) and (Jordi Taltavull, 2019, 41-50).

${ }^{3}$ For a more detailed account of optical theories before 1870 see especially (Darrigol, 2012, 225244), (Whittaker, 1910, 128-169), and (Jordi Taltavull, 2019, 52-55).

${ }^{4}$ There are no biographic references to Sellmeier. Only scant mentions in (Buchwald, 1985, 233), (Darrigol, 2000, 320), and (Darrigol, 2012, 252). In the last two references, he is described as student of Franz Neumann in K'nigsberg and is called Wolfgang. There is some evidence, though, that he could be the author of (Sellmeier, 1890), and therefore be Wilhelm Sellmeier. An analysis of the four papers from 1872 is in (Jordi Taltavull, 2019, 50-60). See also (Jordi Taltavull, 2016, 321-323) for a short introduction to Sellmeier's tetralogy.
} 


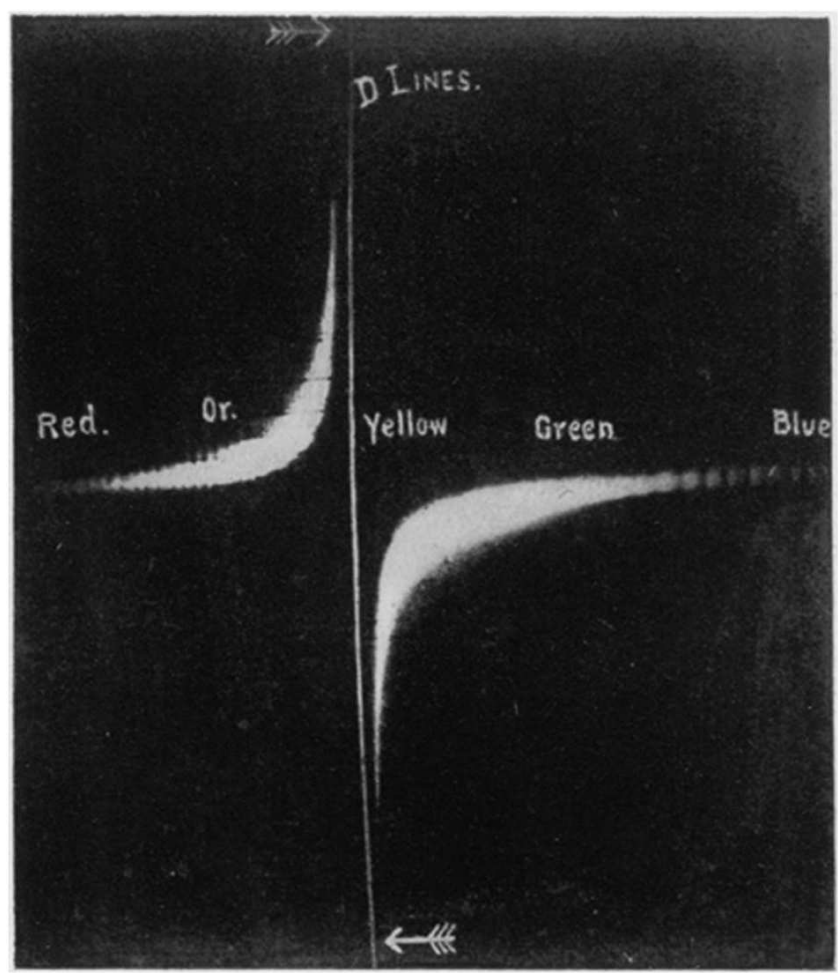

Fig. 1. Representation of anomalous dispersion using the crossed-prism method (Wood, 1902). The discontinuity always takes place at the absorption frequencies of the dispersive material. The dispersing material is not a liquid dye in this case, but sodium vapor. There is no original picture from the early 1870s. To obtain this picture, Wood used the crossedprisms method. It consisted in two prisms with their refractive edges placed perpendicular to each other. Light passing through the first prism should be dispersed in one direction. Each color of the resulting spectrum was dispersed in the perpendicular direction through the second prism. It was easy to observe that, in the neighborhood of the surface colors, at which light was absorbed, the behavior of the index of refraction as a function of the wavelength was not monotonic. Instead, it abruptly increased for increasing wavelength.

and absorption of ether vibrations. In particular, Sellmeier introduced the resonance model for the first time in optics. He assumed that matter consisted of some kind of microscopic particles of matter which vibrated only at specific, characteristic frequencies. Ether waves interacted with these vibrating particles. If the frequency of ether waves coincided with the characteristic frequency of matter particles, these were set into resonance and light was absorbed. For the other frequencies, light was transmitted, albeit with a phase delay that depended on the difference between light frequency and characteristic frequency of matter particles. This gave rise to the different deflection of light depending on its color. Thus, for the first time, matter was not supposed to simply modify the propagation of light, but to act as a tuning fork: at the characteristic frequencies it absorbed light, at the other frequencies, it emitted secondary waves with a phase delay.

According to his calculations, the index of refraction depended on light frequency in the following way: 


$$
n^{2}-1=\sum_{i} \frac{K_{i}}{\nu_{i}^{2}-\nu^{2}}
$$

Here, $n$ denotes the index of refraction, $\nu$ the light frequency, and $\nu_{i}$ and $K_{i}$ are constants of matter that express the characteristic frequencies of matter and $K_{i}$ the "strength" of dispersion at each of these frequencies, respectively. The index of refraction was a summation of the contributions due to all resonance frequencies. At each of them, light was hypothetically absorbed, not dispersed, and the index of refraction displayed a discontinuity. If those resonant frequencies were made to coincide with the surface colors of the liquid dyes used in the experiments, experimental and theoretical data fitted marvelously.

Sellmeier's idea that light and matter interacted through resonance was a turning point in optics. Other physicists, among them so well-known ones as Hermann von Helmholtz (Helmholtz, 1875), Eugene Lommel (Lommel, 1878b), and Eduard Ketteler (Ketteler, 1876; Ketteler, 1879; Ketteler, 1883) embraced Sellmeier's idea in the years afterwards and reformulated it, by introducing different kinds of frictional forces, or by following different mathematical procedures. Nevertheless, they kept the basic idea untouched and they extended the use of the resonance model to all ether-matter interactions in general, such as metallic reflection and phenomena with polarized light (Ketteler, 1885), and fluorescence (Lommel, 1878a). ${ }^{5}$

By the 1890s, the resonance model was so well-established in optics that it was translated into the electromagnetic language, when the electromagnetic theory of light became widely accepted in Europe. Helmholtz (Helmholtz, 1892; Helmholtz, 1893), Hendrik Antoon Lorentz (Lorentz, 1892; Lorentz, 1895), and also Paul Drude (Drude, 1893; Drude, 1894; Drude, 1899; Drude, 1900) devised electromagnetic theories of optical dispersion in the 1890s. The basic idea was that electromagnetic light interacted with electrically charged particles through resonance. When the frequency of the electromagnetic waves coincided with the frequency of those electrically charged particles, matter and ether were also set into resonance. For the other frequencies, electromagnetic waves were transmitted with a phase delay, which was exactly the same as described by the previous theories of dispersion. Not only dispersion, but all magneto-optical phenomena ${ }^{6}$ could be explained on the same grounds, as the result of the interaction between light and moving particles, as presented in Drude's influential textbook on physical optics published in 1900 Lehrbuch der Optik (Drude, 1900). His book was highly influential in setting the agenda for optics in the turn of the century. ${ }^{7}$

With the gradual incorporation of the electrons into physics (Arabatzis, 2006), and the measurement of its charge-to-mass ratio $\frac{e}{m}$, Drude suggested electrons as the moving particles responsible for optical dispersion and most other optical phenomena. To give rise to dispersion, electrons were supposed to perform vibrations. To produce magnetooptical phenomena, electrons presumably performed other kinds of motion. In the case of dispersion, the motion of electrons under the influence of an external

\footnotetext{
${ }^{5}$ Fluorescence is the reemission of light by matter at specific frequencies after prior illumination. See (Jordi Taltavull, 2019, 70-80) for more information about the fine-tuning of Sellmeier's theory by Helmholtz, Ketteler, and Lommel.

${ }^{6}$ These optical phenomena take place when light interacts with a medium that has an external magnetic field applied.

${ }^{7}$ For more information about the electromagnetic translation of the resonance model, see (Jordi Taltavull, 2019, 80-148). See also (Darrigol, 2012, 244-261) and (Darrigol, 2000, 319-332) about Helmholtz and Lorentz. The two last chapters of (Buchwald, 1985) are also devoted to the electromagnetic translation of the resonance model. For a better analysis of Drude's textbook and its impact, see (Jordi Taltavull, 2013b).
} 
electric field $E_{x}$ could be described by the following equation:

$$
m \frac{d^{2} \xi_{i}}{d t}+h_{i} \frac{d \xi_{i}}{d t}+k_{i} \xi_{i}=e E_{x}
$$

The electric field $E_{x}$ was applied in the $x$ direction and followed Maxwell's equations, $\xi_{i}$ was the displacement of the electron $i$ from its equilibrium position in the $x$ direction, $e$ and $m$ were the electric charge and mass of electrons, $h_{i}$ was the damping constant for the electron $i$, and $k_{i}$ was the constant factor of the elastic force. The resonance frequency was $\nu_{i}=\frac{k_{i}}{m}+\frac{h_{i}^{2}}{4 m^{2}}$. The dispersion formula could be derived from the above equation, by assuming wave-like fields. Sellmeier's formula was retrieved by neglecting frictional forces.

The tacit assumption was that the only coupling was the one between electrons and the electric field. Presumably, electrons interacted with light independently from each other, thus no coupling among them was considered. As a matter of fact, ever since Sellmeier, the interaction between ether and microscopic particles of matter was modeled as if particles did not interact with each other. By implicitly assuming such an independent behavior for electrons, Drude concluded that the strength of dispersion $K_{i}$ was proportional to the number of optically active electrons vibrating at each proper frequency $\nu_{i}$. In 1904, (Drude, 1904a; Drude, 1904b) and in the second edition of Lehrbuch der Optik (Drude, 1906), Drude related $K_{i}$ numerically to $\frac{e}{m}$ and to the number $N_{i}$ of electrons having $\nu_{i}$ as the characteristic frequency of natural vibrations. Drude even suggested that the number $N_{i}$ of vibrating electrons pro atom coincided with the valence number:

$$
K_{i}=\frac{4 \pi N_{i} e^{2}}{m}
$$

In this way, optical dispersion and magnetooptical phenomena could be used to explore the microstructure of matter, namely, as a way to determine the characteristic frequencies and the number of optically active electrons in each material. Such an approach is sketched in Drude's Lehrbuch der Optik and Woldemar Voigt's 1908 textbook, Elektro- und Magnetooptik (Voigt, 1908), based on Drude's one, which were the books on optics that physicists, both from the theoretical and experimental quarters, used as reference works. Ladenburg's laboratory was not an exception. In particular, he used Voigt's book as the reference one.

\subsection{Optical dispersion and spectroscopy in the turn of the century}

At the turn of the century, the experimental exploration of anomalous dispersion through a new kind of materials led to novel research questions concerning both experimental and theoretical issues.

Until 1900, anomalous dispersion had been observed practically only around the surface color or surface colors of liquid dyes. Solids, most liquids and gases at normal conditions presented a continuous spectrum in the visible range of frequencies. In these cases, absorption frequencies, around which anomalous dispersion took place, were supposed to lie outside of the visible spectrum and could only be calculated $a$ posteriori, by fitting experimental data into a dispersion formula of Sellmeier's kind. From 1900 onwards, though, a different kind of materials became the focus of interest in experiments of anomalous dispersion, which called for new experimental methods and techniques.

These new materials were gases with spectral lines in the visible spectrum, most significantly metallic vapors, like sodium vapor. The spectral lines were the places in 


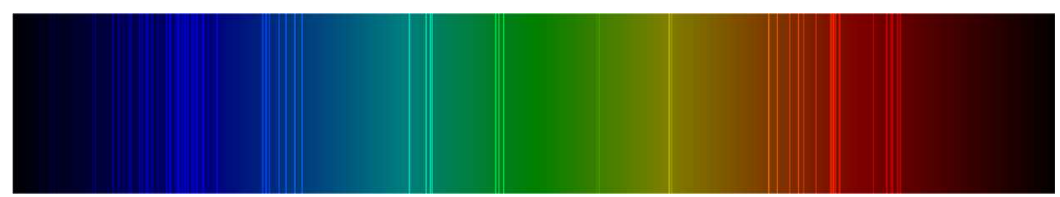

Fig. 2. Image of the sodium absorption spectrum. The lines are the absorption lines, that is, the positions at which light is not transmitted but absorbed by sodium. Source: Wikimedia.

the spectrum at which light was either selectively absorbed or emitted (see Fig. 2). Given the close relation between anomalous dispersion and absorption known for liquid dyes, it was natural to expect that that these materials also exhibited anomalous dispersion in the neighborhood of spectral lines. Yet before 1900 there had been just two isolated attempts to observe anomalous dispersion through sodium vapor, one in 1880 by the already mentioned Kundt (Kundt, 1880), another in 1887 by the German physicist Adolf Winkelmann (Winkelmann, 1887). Both went practically unnoticed.

The interest in measuring anomalous dispersion around the visible spectral lines of gases increased in 1900 due to a novel and bold theory of the Sun proposed by the Dutch physicist Willem Henri Julius in $1900 .^{8}$ Gases with spectral lines in the visible spectrum were part of the solar atmosphere. Julius' idea was that light coming out of the Sun, going through the solar atmosphere, was not only selectively absorbed or emitted by solar gases at exactly the spectral frequencies, but also experienced anomalous dispersion in the neighborhood of theses frequencies (flash spectrum). It was as if the solar atmosphere acted like a prismatic medium, due to hypothetical density inhomogeneities.

Julius was the first in experimentally confirming his theory (Julius, 1900). Yet his was only a first approach, and he urged other physicists to examine anomalous dispersion more carefully through a larger number of this kind of substances to prove his theory. The main challenge was experimental: to adequately prepare the sample so as to produce and measure the desired effects. The American physicist Robert Wood was the first one in taking up Julius' challenge. ${ }^{9}$ He was a gifted experimentalist and months later succeeded in giving sodium the necessary prismatic form to produce the flash spectrum. He accomplished it by heating the metallic sodium of a Bunsenburner at incandescent temperatures just below the surface of a plaster, so that the condensation of the vapor on the cold surface produced the required change of density of the gas that gave rise to the prismatic effects (see Fig. 3). By illuminating the edge of the flame with Sunlight, Wood was able to reproduce the features of the flash spectrum (Wood, 1901; Wood, 1902). Frequencies in the neighborhood were so abnormally dispersed, lied so far displaced from its original direction of propagation, that light of these frequencies went out of the experimental setup (see Fig. 3).

Inspired by Julius' theory, Otto Lummer and Ernst Pringsheim in Berlin, and Hermann Ebert in Munich, performed various experimental analyses of anomalous dispersion through various metallic vapors of the solar atmosphere. Lummer and Pringsheim used sodium, thallium, strontium, calcium, and barium (Lummer, 1903) and Ebert developed a method to give a more stable prismatic structure to potassium (Ebert, 1903).

Once the flash spectrum was experimentally confirmed and solar gases were in the agenda, optical dispersion around spectral lines became interesting for other reasons. In particular, this phenomenon offered the possibility to quantitatively and accurately

\footnotetext{
${ }^{8}$ For more information about Julius and anomalous dispersion, see (Hentschel, 1991). The whole episode about optical dispersion and spectroscopy from an experimental point of view, from Julius to Ladenburg, is told in my dissertation (Jordi Taltavull, 2019, 154-168). It is also summarized in (Jordi Taltavull, 2016, 332-338).

${ }^{9}$ For more biographical details about Wood, including his optical research, see (Seabrook, 1941).
} 


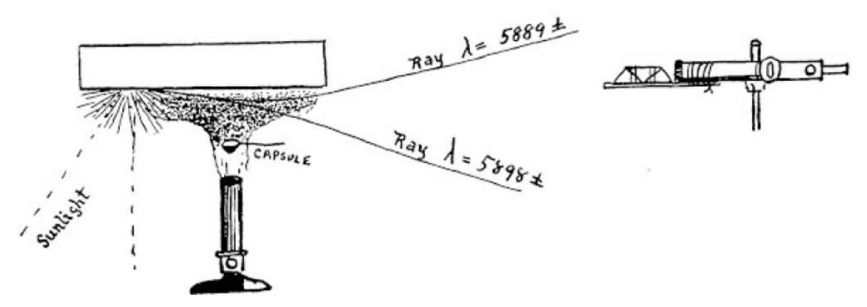

Fig. 3. Reproduction of the flash spectrum by Wood (Wood, 1901). Two rays are abruptly dispersed.

test Sellmeier's formula in the neighborhood of absorption lines. So far, it had not been possible to measure the index of refraction in the neighborhood of absorption frequencies with enough precision to properly check Sellmeier's formula (Wood, 1904). Ever since 1871 it was clear that liquid dyes exhibited anomalous dispersion around their surface colors, yet surface colors were broad absorption bands, and therefore the fitting of experimental data to Sellmeier's formula was difficult to do precisely. Spectral lines were, on the other hand, sharp dark lines. The use of liquid dyes had another drawback: it was not possible to obtain numerical values of the index of refraction very close to the resonance frequencies, since there did not exist sources of monochromatic light whose frequencies were close enough to the surface colors at various frequencies. Due to the position of spectral lines, it was possible to approach the spectral lines of one solar gas by using other solar gases as sources of monochromatic light. These emitted light exactly at the frequencies at which they also absorbed light selectively. Thus the measurement of anomalous dispersion around spectral lines had crucial advantages in comparison with the measurement of anomalous dispersion around the surface colors of liquid dyes.

Wood devoted years to obtain good quantitative measurements of anomalous dispersion around the $\mathrm{D}$ spectral lines of sodium vapor. The first challenge was to build a prism of sodium vapor that remained stable for long time and whose equivalent angle could be calculated. Then he measured the index of refraction around two of the spectral lines of sodium vapor $D_{1}$ and $D_{2}$, using two methods (Wood, 1904). First of all he made light pass through the sodium prism and decomposed it into the color spectrum through a spectroscope of high precision. An alternative method was to use the interferometer. Interferometers merged two different light sources to create an interference pattern. A change in the pathway of one of the two beams implied a phase delay of one beam with respect to the other, which resulted into a displacement of the pattern of interference fringes. In particular, Wood made two monochromatic beams interfere. When one of the two beams was made to pass through the prism of sodium vapor, the interference pattern was displaced. The number of interference fringes that were displaced and disappeared from the pattern was directly proportional to the phase delay experienced by the beam that had passed through the prismatic medium. Thus the displacement of the interference lines was a measure of the index of refraction of sodium vapor at the frequency of the monochromatic source of light used in the experiment.

Somewhat paradoxically, despite the unprecedented high precision of his results, Wood's mathematical analysis was rather disappointing. Since he measured anomalous dispersion around two spectral lines, instead of one, he could have used a two-term Sellmeier's formula, each term corresponding to one spectral line. Nevertheless, he limited his calculations to a single-term formula, by arguing that the two frequencies were very close to each other, the experimental values of the index of refraction in the region between the two frequencies were less accurate than the 
values outside of this region, and therefore not much could be learned from using a two-term Sellmeier's formula. ${ }^{10}$

Yet not all physicists had the same opinion. According to the Polish physicist Ladislas Natanson, the use of a two-term Sellmeier's formula offered the unprecedented possibility to check the microscopic interpretation of optical dispersion, an opportunity that Wood missed (Natanson, 1907). As Natanson observed, all theories of dispersion were so far based on what he called the "simplifying hypothesis": atoms and molecules contained only one kind of resonators. The model was then generalized to various resonance frequencies by simply adding as many summation terms as there were resonance frequencies to the molecule, without ever reflecting on the possible role of complex molecular structures or the interaction between the various kinds of resonators. This interpretation could be challenged by a quantitative analysis of dispersion in the neighborhood of more than one spectral line, for this meant that more than one kind of resonator coexisted within the same atom.

For this reason Natanson took Wood's experimental data and fitted them into a two-term Sellmeier's formula. The characteristic frequencies of Sellmeier's formula were supposed to coincide with the spectral frequencies. A value of $K_{i}$ for each spectral line could be obtained from experimental data. It turned out that $K_{2}$ was considerably larger than $K_{1}$. Given Drude's proportionality relation between $K_{i}$ and number $N_{i}$ of optically active electrons vibrating at the corresponding frequency $\nu_{i}$, Natanson concluded that the number of electrons vibrating at the spectral frequency $D_{2}$ should be considerably larger than the number of electrons vibrating at the spectral frequency $D_{1}$. The new question was: why were there more electrons resonating at the frequency $D_{2}$ than at the frequency $D_{1}$ ? More generally, how did multiple resonators behave within complex atomic and molecular structures? The "simplifying hypothesis" and the simple proportionality hypothesis between optical parameters and the number of microscopic resonators did not suffice to answer these questions.

\section{Rudolf Ladenburg and optical dispersion before the WWI}

\subsection{Ladenburg and his first approach to anomalous dispersion in 1908}

Both experimental and interpretative challenges of anomalous dispersion around spectral lines were taken in Ladenburg's laboratory from 1908 onwards. ${ }^{11}$

Ladenburg was born into a family of scientists. ${ }^{12}$ His father Albert was an eminent chemist at the University of Breslau from 1886 until he died in 1911, and his brother Erich also made a scientific career at the same University until his premature death in $1908,{ }^{13}$ where he did significative research on the photoelectric effect. Ladenburg followed the same scientific path, thus from 1900 onwards he studied physics in Heidelberg, Breslau, and Munich, where he obtained his PhD in 1906, and thereafter he

\footnotetext{
${ }^{10}$ The resonance frequency $\nu_{0}$ he used was the mean value of the two spectral frequencies.

${ }^{11}$ See chapter three of (Jordi Taltavull, 2019).

${ }^{12}$ To learn about Ladenburg's renowned Jewish family, see (Waldeck, 1920, 67-84). In (Kopfermann, 1952) and (Shenstone, 1973) we find few biographical details. About Ladenburg's emigration to USA and national socialism, see (Hentschel, 1996). In (Born, 1975) we find short references to Ladenburg, since Max Born and Ladenburg were close friends. Born's autobiographical notes will be quoted later.

${ }^{13}$ This sad episode is told in Albert Ladenburg's memories (A. Ladenburg, 1912, 113-114,152153): in Albert Ladenburg's memories we find few references to his second son Rudolf, apart from the fact that he accompanied his father when his mother died scarcely 6 months after the premature death of the eldest brother Erich due to a boat accident in the Mügel lake (A. Ladenburg, 1912, 6-7). Rudolf was the only son out of three that survived his father and his mother. The youngest one, Kurt, died at the age of 12 in 1901 (A. Ladenburg, 1912, 114,134-138).
} 
came back to Breslau, where he worked as Privatdozent and then extraordinary professor until 1924. During WWI Ladenburg was first member of the cavalry and then sent to Berlin to carry out research on ballistics and acoustics (Born, 1975). In 1924 he was appointed director of the physics department of the Kaiser Wilhelm Institute for physical chemistry and electrochemistry of Berlin, and in 1931 he emigrated to the USA, where he was invited professor at Princeton University.

Although his early research in Munich concerned viscosity of liquids, as early as 1908 he became interested in optics, a field in which he was active until he left Germany in 1931. It is in the tradition of optics motivated by astronomical interests that we must situate Ladenburg's first optical experiments. The first optical phenomenon that caught his attention had also astronomical implications, like the flash spectrum. It was the reversal of spectral lines, i.e. the phenomenon according to which gases emitting light at specific frequencies also absorbed light at the same frequencies. The phenomenon was of interest for astronomers since the American astronomer William W. Campbell had formulated a theory of stars according to which light emitted by a gas in the inner layers of a stellar atmosphere would be partially absorbed by more peripheral layers of the same atmospheric gas (Campbell, 1895). In other words, emission lines were reversed. Furthermore, the quantity of absorption, thus the strength of the reversal, changed for each spectral line. This would explain a curious pattern in the spectrum of Orion Nebula detected by Campbell in 1895: the luminosity of the spectral lines of the monoatomic gas of hydrogen seemed to decrease for larger wavelengths. Kirchhoff's law provided the theoretical framework, yet this phenomenon was very difficult to confirm experimentally: how could one prove, on the basis of spectral analysis, that both emission and absorption had taken place if the two occurred simultaneously at the same frequencies?

Ladenburg's 1908 experimental confirmation of the reversal of spectral lines is interesting in our context because it was here that Ladenburg devised an experimental method to produce anomalous dispersion and learned how to manipulate another gas of astronomical interest with spectral lines in the visible range of frequencies: monoatomic gas of hydrogen (Ladenburg, 1908). The experimental setup used to produce the reversal of the spectral lines of the monoatomic gas of hydrogen was the same as the one that produced anomalous dispersion around the spectral lines of the same gas. Ladenburg had to confront two major challenges in devising the experiment: first, how to generate the right conditions for the gas to emit and absorb light at spectral lines. Hydrogen in normal conditions is a molecular gas with no spectral line in the visible spectrum. Instead, the kind of hydrogen gas present in stellar atmospheres was atomic gas and emitted at the well-known spectral lines of the Balmer series. To obtain such a gas, Ladenburg had to excite normal hydrogen gas electrically, by producing an electric discharge. Under these conditions, hydrogen emitted at the well-known frequencies of the Balmer series.

The second challenge was to invent a way to experimentally prove that monoatomic hydrogen emitted and simultaneously absorbed light at the same frequencies. This was not an easy task, for what was observed directly was an emission spectrum. The key for a successful and convincing production of the reversal of spectral lines in hydrogen laid in the appropriate density and pressure of gas, so that the emission line was spread over a small range of frequencies, whereas the absorption appeared as a dark line in the middle. ${ }^{14}$ His experimental procedure was the following. He prepared two capillary tubes of the same length, filled with hydrogen gas and connected in series to one single induction-coil circuit. When the circuit was

\footnotetext{
${ }^{14}$ In 1907 the German physicist Alexander Pflüger associated the previous experimental failures in producing the reversal of spectral lines with hydrogen to a very low pressure of the gas (Pflüger, 1907). One year later, Ladenburg came up with a suitable experimental device and yielded the first quantitative account of the phenomenon (Ladenburg, 1908).
} 


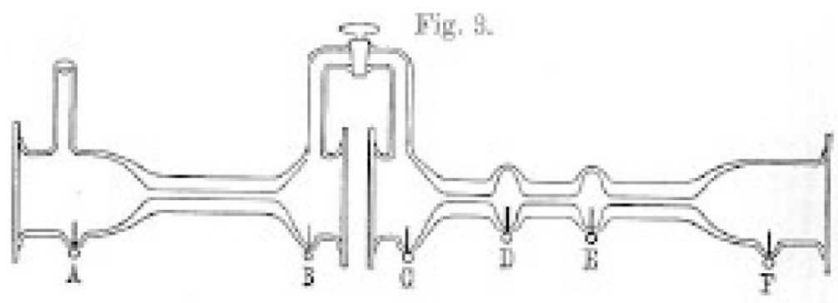

Fig. 4. The emission and absorptions tubes in series. The second one has the possibility to be electrically excited at different lengths (Ladenburg, 1908, 553).

connected, the gas emitted radiation simultaneously in both tubes. As it is shown in Figure 4, the length of one tube was fixed $(\mathrm{AB})$, while the other tube was especially constructed such that one could chose the length of the gas column to be electrically excited (CDEF). Parallel to the tubes a spectrometer was placed, on the screen of which one could analyze the spectrum of the excited gas. By means of this device Ladenburg could experiment with different densities and pressures of the gas, until he achieved the desired patterns: very unsharp emission lines, in the center of which dark lines appeared due to absorption. In this way, he reproduced the reversal of spectral lines and proved that emission and absorption took place at the same frequencies.

Exactly the same setup served to produce anomalous dispersion around the spectral lines of the monoatomic gas of hydrogen. Since the emission of light by atomic hydrogen spread over a broader range of frequencies than absorption, one observed a colored spectrum in the neighborhood of the absorption line. Such colored spectra resulted from the transmission of light through the monoatomic gas of hydrogen close to the absorption lines. At these frequencies light was absorbed, instead of being transmitted. But this is exactly the definition of anomalous dispersion and Ladenburg immediately realized that. Indeed, his following paper was about anomalous dispersion around the spectral lines of the monoatomic gas of hydrogen. which he produced by means of the device described above (Ladenburg, 1908).

\subsection{A new concept emerges: "dispersion electrons"}

Ladenburg's interest in dispersion was not confined to its experimental reproduction. In contrast to Wood, Ladenburg soon became deeply concerned about interpretative issues, in particular, how to use experimental data of dispersion to obtain information about the microstructure of matter. As a matter of fact, Ladenburg was not only a very skilled experimentalist, but also an able theorist. He did not follow complicated mathematical developments, but he was very good at using basic mathematical formulas and theoretical ideas for the sake of interpreting experimental results.

Ladenburg was not alone in performing dispersion experiments and discussing the results between 1908 and 1911. Stanislau Loria joined Ladenburg's laboratory, as an assistant, in 1908. Loria was a former student of Ladislas Natanson in Cracow and one of his greatest admirers, as Max Born stressed in (Born, 1975, 182). Thus it would not be surprising that Loria knew of Drude's dispersion theory and was even aware of the tricky relation between optical parameters and the number of electrons vibrating at the hypothetical resonance frequencies. How to relate experimental results to the microstructure of matter became indeed a central topic in Ladenburg's and Loria's investigations about optical dispersion in the following three years.

Ladenburg's laboratory also received regular visits from two theoretical physicists, then just arrived at Breslau: Max Born and Fritz Reiche. Both enjoyed discussing 


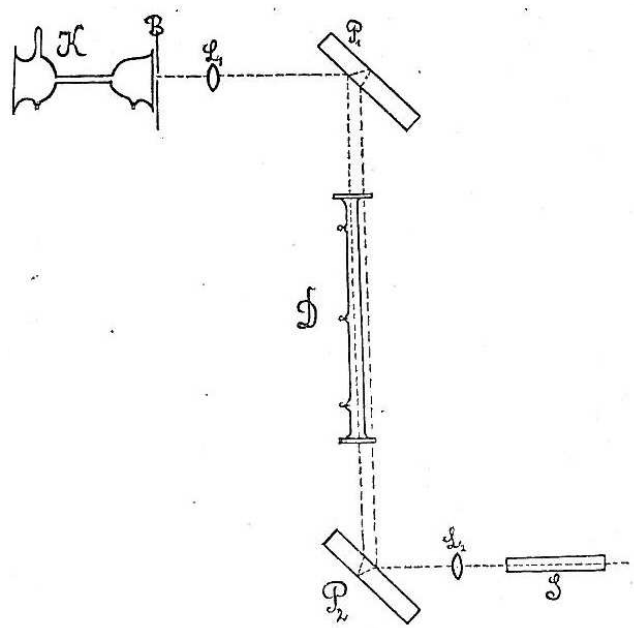

Fig. 5. Ladenburg and Loria's experimental setup. E and D are the two separated capillary tubes. $\mathrm{E}$ is placed before the interferometer $\left(P_{1}\right.$ and $\left.P_{2}\right)$ and $\mathrm{D}$ in one of the two interferometer pathways. $\mathrm{S}$ is the spectrometer. Image from (Ladenburg and Loria, 1908b, $875)$.

about what happened in Ladenburg's dark laboratory. In his autobiography, Born illustrated those moments with the following words:

Sein -of Ladenburg- verdunkelter Raum, in dem einige Kapillarrohre in einem rötlichen Licht glühten, faszinierte mich wie die Höhle eines Zauberers. Er hatte eine unheimliche Geschicklichkeit im Glasblasen und anderen Techniken, die ich mit wenig Erfolg von ihm zu lernen suchte. Während er mit seinen Röhren und Drähten arbeitete oder sein Spektroskop adjustiere, kommentierte Loria ununterbrochen die Theorie zu diesem Phänomen -optical dispersion-, wobei ihn Reiche manchmal mit einem sarkastischen "Ach, das ist doch alles Quatsch" unterbrach. Dann flammte eine wilde Diskussion auf (Born, 1975, 183).

In this lively context Ladenburg and Loria succeeded in obtaining rather accurate experimental results and in pinpointing a key interpretative problem. The first step was to obtain satisfactory measurements. Ladenburg and Loria did so in 1908 for anomalous dispersion around the spectral line $H_{\alpha}$ of the electrically excited hydrogen gas. This was the brightest spectral line of the famous Balmer series, which had a red color. To excite the gas electrically, Ladenburg and Loria used the above-mentioned system, namely, the two capillary tubes connected in series to an induction-coil system and filled with hydrogen gas (Ladenburg and Loria, 1908a). ${ }^{15}$ To obtain measurements, they separated the two tubes and combined them with an interferometer and a spectrometer. One tube acted as a source of light and was placed before the interferometer. The other tube was to absorb and disperse light and it was arranged along one of the paths of the interferometer (see Fig. 5).

When the two tubes were excited simultaneously, both emitted and absorbed light at the same spectral frequencies. Light emitted by the source ( $\mathrm{K}$ in Fig. 5) was made to pass through the interferometer, and there it was divided into two rays that followed two parallel paths. The ray traveling along the path with the capillary tube (D in Fig. 5) was obviously delayed with respect to the light traveling along

\footnotetext{
${ }^{15}$ Reprinted in (Ladenburg and Loria, 1908b).
} 


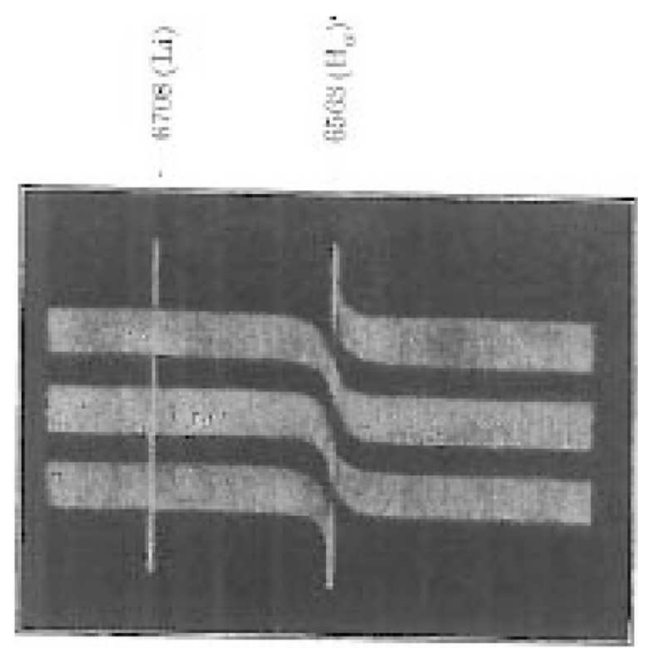

Fig. 6. Interference pattern of anomalous dispersion around the spectral line $H_{\alpha}$ (Ladenburg and Loria, 1908b, 861).

the other path. At the outset of the interferometer, the two light rays were made to interfere. The interference pattern was decomposed into the whole spectrum of frequencies within the spectrometer ( $\mathrm{S}$ in Fig. 5). The resulting interference pattern is shown in Figure $6 .^{16}$

The phase delay of light when passing through the second capillary tube resulted in a displacement of the interference fringes with respect to interference pattern that was observed when no tube was placed or no hydrogen was electrically excited. In turn, the delay of light was directly related to the index of refraction $n$ of the substance delaying light. Dispersion amounted precisely to the dependence of $n$ on light frequency $\nu$. Thus to numerically measure dispersion around the spectral lines of monoatomic hydrogen gas, Ladenburg and Loria had to calculate the displacement of interference fringes caused by the hydrogen gas of tube $D$ at several positions of the spectrum - the frequency was given by the grating of the spectrometer. Eventually, by fitting these numerical data into Sellmeier's formula, it was possible to compute a value for the parameter $K_{\alpha}$ (see formula (1)). The value of $\nu_{\alpha}$ was obviously determined by the frequency of the spectral line, $H_{\alpha}$.

The goal of Ladenburg and Loria was not simply the confirmation of Sellmeier's formula. In fact, they simply took the formula for granted. More importantly, Ladenburg and Loria wanted to use the formula to obtain more detailed information about the underlying microstructure of matter. More specifically, they calculated the number $N_{\alpha}$ of electrons vibrating hypothetically at the frequency $H_{\alpha}$, by using Drude's assumption that $K_{\alpha}$ and $N_{\alpha}$ were directly proportional. Finally, by taking

\footnotetext{
${ }^{16}$ This experimental setup was based on Luigi Puccianti's interferometric method. Puccianti's was an Italian physicist, professor at the University of Pisa (Polvani, 1952). Julius' theory of the flash spectrum had also called his attention, although he did not direct his efforts to construct gas prisms, as Wood, Lummer, Pringsheim, and Ebert did. Puccianti was interested in both visualizing and measuring the index of refraction of metallic vapors around their spectral lines. To do so, in 1905 he suggested a new experimental procedure that enabled both qualitative and quantitative accounts of anomalous dispersion (Puccianti, 1905). The crux of Puccianti's procedure lied in the combined use of an interferometer and a spectroscope, in the way Ladenburg and Loria's experimental setup worked. As a matter of fact, Puccianti's apparatus resembled very much one he invented in 1901 to measure the refractive index of the oxyhemoglobin (Puccianti, 1901). For a more detailed account, see (Jordi Taltavull, 2019, 161-162).
} 
into account the number of atoms present in the sample, ${ }^{17}$ they reached the puzzling conclusion that only one electron out of 50,000 atoms of hydrogen would participate in the optical process.

There was still another issue. Electrically excited hydrogen gas was characterized by other spectral frequencies besides $H_{\alpha}$, most importantly $H_{\beta}$ and $H_{\gamma} . H_{\beta}$ and $H_{\gamma}$ were of higher frequency than $H_{\alpha}$, respectively. Both had a bluish color and were less bright than the $H_{\alpha}$. Ladenburg and Loria did not achieve numerical results for $n$ around these frequencies, but they nevertheless could qualitatively observe that the effect of anomalous dispersion was less strong around $H_{\beta}$ and $H_{\gamma}$ than around $H_{\alpha}$. From this observation they guessed that an even lower number of electrons per atom was vibrating at the corresponding resonance frequencies.

Ladenburg and Loria had encountered the first pitfall of former theories of dispersion. The very low proportion of vibrating electrons per atom completely undermined Drude's assumption that the number of vibrating electrons per atom coincided with the valence number. More fundamentally, the question arose as to why only a very low number of electrons participated in dispersion. Moreover, as Ladenburg and Loria remarked, theirs was not the only case in which a very low number of electrons appeared to play a role: in experimental explorations of magnetorotation of light polarization, another optical phenomenon observed in the neighborhood of the spectral lines of sodium vapor, carried out in Lorentz's laboratory in Leiden ${ }^{18}$ and Voigt's laboratory in Göttingen (Geiger, 1907), only 1 electron per 200 molecules was calculated to be optically active. The magnetorotation of light polarization was the dependency of the angle of rotation of light polarization $\chi$ on light frequency $\nu$ when light passed through a substance with an external magnetic field applied. As in the case of dispersion, an anomalous and discontinuous behavior of $\chi(\nu)$ was observed in the neighborhood of the same frequencies around which dispersion $n(\nu)$ was anomalous. ${ }^{19}$

At that point, the interpretative problem did not only concern optical dispersion, but it was an interpretative problem that concerned more generally the way in which optical parameters were related to the microstructure of matter (to the number of optically active resonating electrons) by means of a simple proportionality relation. In order to better refer to this problem, Ladenburg and Loria introduced a new concept, "dispersion electrons" (Ladenburg and Loria, 1908a, 866), which became central to Ladenburg's and Loria's investigations in the following years. These were the electrons optically active at each resonance frequency, which were responsible not only for dispersion but also for any other kind of optical phenomena implying light-matter interaction, like the magnetorotation of light polarization, among others.

\subsection{The problem of "dispersion electrons": 1909-1911}

In the following two years, Ladenburg's and Loria's investigative program revolved around how to interpret the number of dispersion electrons. The low number of dispersion electrons was not the only puzzling result. Ladenburg and Loria measured a persistent asymmetry in the number of dispersion electrons vibrating at different spectral lines, for which previous theories of dispersion had no explanation either.

\footnotetext{
${ }^{17}$ Ladenburg's and Loria's experiments had a big advantage with respect to Wood's in that the quantity of gas contained in the tubes was known. Thus by having control over the temperature one could immediately guess the number of atoms of hydrogen being excited.

${ }^{18}$ Voigt refers to one dissertation written by one of Lorentz's students, whose surname is Hallo. Voigt thanks Lorentz for having let him read Hallo's dissertation (Voigt, 1908, 141-144).

${ }^{19}$ To learn more about the relationship between the magnetorotation of light polarization and anomalous dispersion from both theoretical and experimental points of view, see (Jordi Taltavull, 2019, 177-183).
} 
New experiments and calculations were carried out in the period between 1909 and 1911 by Ladenburg and Loria, who divided their efforts in this period. Loria repeated and improved Wood's measurements and calculations with sodium. Ladenburg concentrated on hydrogen gas and its other two spectral frequencies $H_{\beta}$ and $H_{\gamma}$.

In 1909, Loria published a paper with the first calculation based on dispersion experiments, of the number of dispersion electrons per sodium atom vibrating at each one of the spectral frequencies $D_{1}$ and $D_{2}$ (Loria, 1909). Previous calculations of the number of dispersion electrons within sodium atoms had been obtained from experiments of magnetorotation of light polarization. To achieve his goal, Loria made use of the interferometric method to obtain accurate measurements of the index of refraction around the two spectral lines of sodium $D_{1}$ and $D_{2}$ explained above (see Fig. 5). In contrast to Wood, Loria's experimental setup allowed him to calculate the amount of sodium atoms dispersing light, because he worked at a temperature for which it was possible to use previous experimental results relating temperature to sodium gas density (Jewett, 1902). ${ }^{20}$ This piece of information was necessary in order to calculate the ratio of dispersion electrons per atom. For the numerical calculations, Loria used a two-term Sellmeier's formula centered at the spectral frequencies $D_{1}$ and $D_{2}$.

Loria's result was twofold: first of all, he confirmed once again the very low number of dispersion electrons per atom participating in dispersion, specifically 1 electron per 200 atoms vibrating at the $D_{1}$ spectral frequency, and 3 electrons per 400 atoms at the frequency $D_{2}$. The first result fully coincided with results obtained from experiments of magnetorotation of light polarization. Secondly, he corroborated an asymmetry between the number of dispersion electrons $N_{1}$ and $N_{2}$ resonating at the $D_{1}$ and at $D_{2}$ frequencies. In particular, $N_{2} / N_{1}=1.3$, which was slightly different from the ratio $N_{2} / N_{1}=2$ previously obtained by Geiger on the basis of experimental data of magnetorotation of light polarization.

The evidence for such an asymmetry of dispersion electrons between the two lines of the sodium spectrum was deemed by Loria as a key point for further research. So far, all experiments with sodium vapor had displayed such an asymmetry, either qualitatively -Wood's experiments with dispersion (Wood, 1904)-, or quantitatively -Geiger's data (Geiger, 1907)-. Now it appeared to be time to explain why the asymmetry existed. It was clear to Loria that it reflected some deep relationships within the structure of matter: "Man kann nicht leugnen, daß diese übereinstimmenden Merkmale auf eine tiefere liegende Beziehung hinweisen, an deren Begründung jedoch man sich heutzutage nur mit größer Vorsicht heranwagen kann" (Loria, 1909, 254).

The strong asymmetry in the number of dispersion electrons vibrating at different spectral lines was corroborated by Ladenburg in the same years. In particular, Ladenburg concentrated on the monoatomic gas of hydrogen, with which he performed experiments of both dispersion (Ladenburg, 1911) and magnetorotation of light polarization (Ladenburg, 1909a). The same extremely low number of dispersion electrons was confirmed again: 1 electron per 50000 atoms vibrating at the $H_{\alpha}$ Balmer frequency. A strong asymmetry in the number of dispersion electrons hypothetically resonating at the different spectral frequencies of the Balmer series was also confirmed: from $H_{\alpha}$ to $H_{\gamma}$, the number $N_{i}$ of dispersion electrons decreased systematically, being $N_{H_{\beta}}=\frac{1}{4} N_{H_{\alpha}}$. Such an asymmetry can be appreciated in Figure 7 . Since anomalous dispersion in the neighborhood of $H_{\gamma}$ was very faint, it was difficult to calculate $N_{H_{\gamma}}$, yet it was clear that this number was much smaller than for the other lines.

\footnotetext{
${ }^{20}$ Upon knowing the temperature of the sodium vapor one could immediately guess its density, if the temperature was between 360 and 420 degrees, since experiments were available relating temperature to density in the above-mentioned range of temperatures (Jewett, 1902). Since Wood's experiments were carried out at 644 degrees, this information was useless to him.
} 


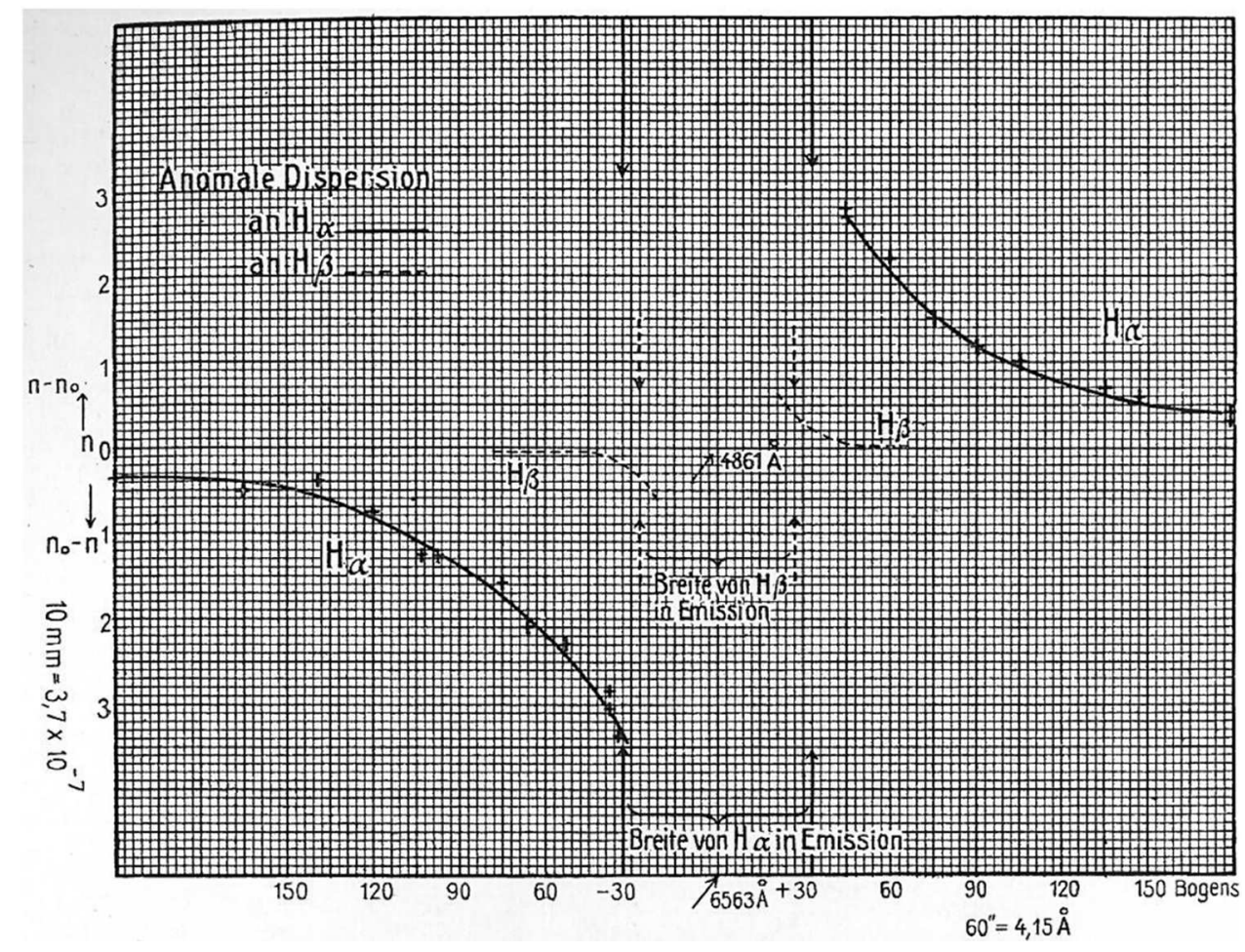

Fig. 7. Ladenburg's experimental data of anomalous dispersion around $H_{\alpha}$ and $H_{\beta}$ of the electrically excited hydrogen gas (Ladenburg, 1911, 11).

Loria and Ladenburg were not the only ones pointing out to this asymmetry in dispersion electrons between different spectral lines. The British experimentalist P. V. Bevan arrived at the same conclusion by measuring anomalous dispersion through potassium vapor (Bevan, 1910). He also obtained an extremely low number of dispersion electrons (only 1 electron per 2000 molecules resonating at the violet spectral lines of potassium vapor), and detected a sharp decrease of $N_{i}$ for decreasing wavelength as well (Fig. 8). For example, the ratio of dispersion electrons resonating at the red lines of potassium vapor to the number of dispersion electrons vibrating at the violet lines was about $1 / 700$.

Altogether, the problem of dispersion electrons was not limited to the low number of resonating electrons per atom, ${ }^{21}$ but it encompassed another aspect, which eventually became much more important than the first one: the asymmetry in the number of dispersion electrons vibrating at the different spectral frequencies. According to Loria, the asymmetry revealed some unknown deep feature of the inner structure of atoms and molecules. According to Bevan, instead, electrons in metallic vapors could form different temporary combinations with the same atom, so that different arrangements of the same chemical substance coexisted in the same sample. Each

\footnotetext{
${ }^{21}$ In (Duncan, 2007a, 583), the low number of dispersion electrons per atom seems to be the only problem.
} 


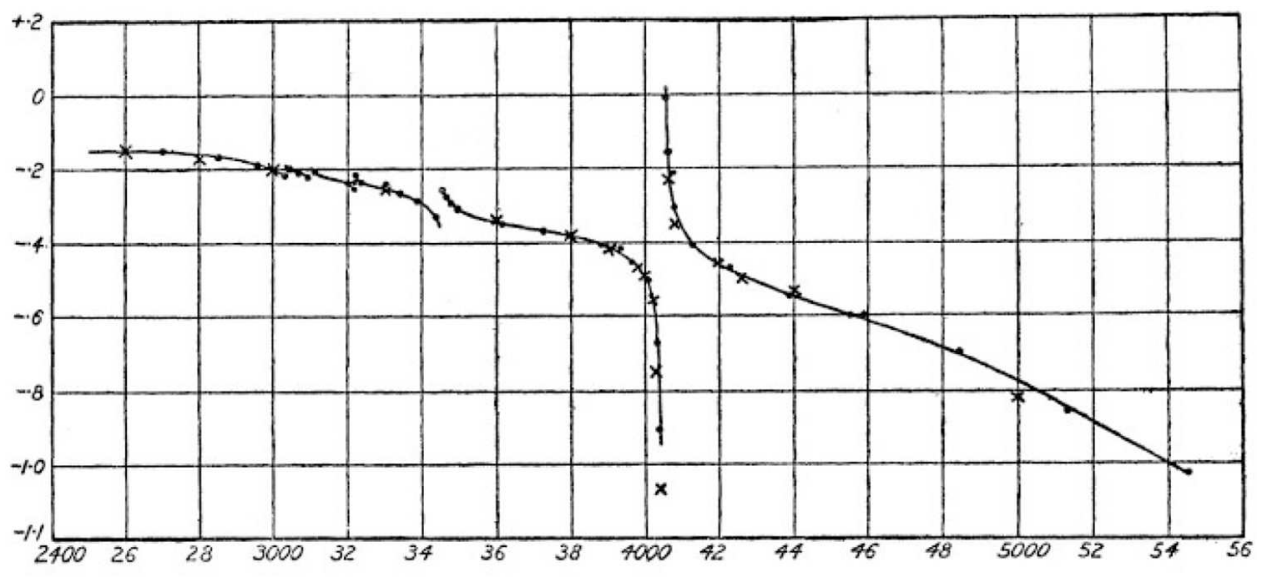

Fic. 7.-Abscissa is wave-length; ordinate is deviation.

Fig. 8. Bevan's experimental data of anomalous dispersion around the spectral lines of potassium vapor (Bevan, 1910, 217).

arrangement gave rise to one spectral line, thus it was the number of these arrangements (only few atoms in each case) what explained the low value of $N_{i} \cdot{ }^{22}$ Ladenburg's line of thought went in Loria's direction: the number of dispersion electrons did not correspond to the number of individual entities vibrating at certain frequencies, but to some other property of atomic and molecular structures in their way to interact, as a whole, with light. To understand better Ladenburg's approach, it is necessary to understand the purely theoretical challenges that Drude's dispersion theory faced in the case of substances with spectral lines in the visible spectrum, which were also discussed in Breslau in the same period of time.

\subsection{Dispersion and spectroscopy from a theoretical point of view}

The simple hypothesis that resonant frequencies were due to dispersion electrons vibrating independently from other electrons within atoms and molecules was not only challenged in laboratories, but also in theoretical accounts. The interplay of optical dispersion and spectroscopy became a theoretical problem in itself from 1908 onwards.

The British mathematician George A. Schott (Schott, 1908) and, more importantly, the Breslau physicist Clemens Schaefer (Schaefer, 1909a; Schaefer, 1909b; Schaefer, 1910) worked on specific theories of optical dispersion around spectral lines between 1908 and 1910 .

In 1908, Schott pointed out very significant difficulties of Drude's theory when it was applied to anomalous dispersion around spectral lines. First of all, Schott demonstrated that Drude's formula of $n(\nu)$ did not converge for infinite terms of the summation. More specifically, Schott showed that $n(\nu)$ was divergent for the case of the Balmer series, whose wavelengths $\lambda_{i}$ were characterized by the following expression, $\lambda_{i}=\lambda_{\infty} \frac{m^{2}}{m^{2}-4}$, with $m=3, \ldots, \infty . \lambda_{\infty}$ was a constant (Schott, 1908).

\footnotetext{
${ }^{22}$ Bevan's explanation was in full agreement with Joseph J. Thomson's explanation of the Zeeman effect, according to which the splitting of spectral lines was due to the different temporary combinations of ions with atoms, instead of some inner property of atoms (Jordi Taltavull, 2019, 187-189).
} 
If the series was not infinite, but only contained a very high number of terms, Drude's theory presented problems as well. Schott resorted again to the Balmer series, of which 29 lines had already been experimentally detected. By assuming that only one electron per atom was optically active, all having three vibrational degrees of freedom, the theoretical value of $n^{2}-1$ turned out to be much higher than the experimental value. Schott reached the following conclusion:

Die Hypothese, daß Serienlinien durch kleine Elektronenschwingungen erzeugt werden, ist in grellem Widerspruch mit der Erfahrungstatsache des gleichzeitigen Bestehens von Serien und von einem endlichen Brechungskoeffizienten für lange Wellen (Schott, 1908, 215-216).

And he finished with this strong statement about Drude's theory: "Sie muß deswegen aufgegeben werden" (Schott, 1908, 216).

Schaefer suggested a very interesting solution to Schott's problem (Schaefer, 1909a; Schaefer, 1909b). Schaefer was also working at the University of Breslau, where he habilitated in 1903 and became ordinary professor of physics in 1910 (Bergmann, 1958). According to him, the main problem of Drude's theory was that interactions among electrons were completely ignored. Instead of an equation of the same kind as equation (2), Schaefer considered a motion equation of the following kind:

$$
m_{i} \frac{d^{2} \xi_{i}}{d t^{2}}+f_{i}\left(\xi_{1}, \xi_{2}, \xi_{3} \ldots \xi_{s}\right)=e_{i} E_{x}
$$

The main difference between equations (2) and (4), after neglecting frictional forces, was the new term $f_{i}\left(\xi_{1}, \xi_{2}, \xi_{3} \ldots \xi_{s}\right)$, which substituted the old term $k \xi$ of equation (2) corresponding to the elastic restoring force applied to each particle $i$. The new term coupled the motion of the particle $i$ with the motion of the other $s-1$ particles. Hence electrons were not independent of each other, but formed "systems" of electrons that interacted with light as a whole. This made a big difference compared with all theories of optical dispersion displayed hitherto since 1872. In Schaefer's framework, the simple identification of natural vibrations of individual electrons with resonance frequencies was only one possible approximation, which was uniquely valid when the "systems" had very few degrees of freedom, and the coupling between electrons could therefore be neglected. In fact, this approximation worked well for solids, liquids and gases having normal dispersion, and coloring liquids with only one proper frequency in the visible. Instead, the issue of the coupling was relevant in the context of anomalous dispersion around multiple spectral lines.

To tackle the problem mathematically, Schaefer decided to approximate the "system" composed of $s$ electrons to a continuous medium with infinite degrees of freedom, so that the entire mass and charge of the continuous system remained the same as the one with $s$ particles. ${ }^{23}$ More specifically, Schaefer modeled the atomic systems as if they were continuous strings with finite mass and charge and infinite modes of vibration (harmonics). This assumption obviously implied that electrons were not independent of each other, but that some kind of coupling had to be taken into account. Resonance frequencies did not correspond to the natural vibrations of individual electrons, but to the resonance vibrations of the string as a whole. Thus the

\footnotetext{
${ }^{23}$ To approximate a discontinuous system with finite degrees of freedom (like a finite number of particles) to a continuous system with infinite degrees of freedom (like a continuous string) was a commonplace approximation when dealing with the problem of a vibrating string. As accounted by Schafer, this approximation was called "Rayleigh's Principle". For example, one could consider a continuous string as if it was composed of a finite number of massive points or one could model a string as a finite series of small pendula close to each other (Schaefer, 1909a, 424-425).
} 
problem was reduced to solving the problem of forced vibrations of a string under the influence of an external electric field $E_{x}$ :

$$
\rho \frac{\partial^{2} \xi(x, t)}{\partial t^{2}}-p \frac{\partial^{2} \xi(x, t)}{\partial x^{2}}=e^{\prime} E_{x}
$$

$\xi(x, t)$ was the displacement of each point $x$ of the string at a certain moment $t, \rho$ was the mass density of the string, $e^{\prime}$ was the charge density, and $p$ was a constant factor that depended on the string. If the length of the string was 1 , then the boundary conditions specified that $\xi_{x=0}=0$ and $\xi_{x=1}=0$. As a result, the displacement of each point of the string was obtained as a superposition of the infinite solutions of the nonforced case, namely, of the infinite proper vibrations of the string for non-electric field applied. ${ }^{24}$

Eventually, Schaefer arrived at a dispersion formula for $N^{\prime}$ "systems" that was structurally the same as Drude's, yet with fundamental differences concerning the parameters and their physical interpretation. Schaefer's formula was the following one:

$$
n^{2}-1=4 \pi \sum \frac{N^{\prime} M_{i}}{\nu_{i}^{2}-\nu^{2}}
$$

$M_{i}$ was a new parameter associated to each frequency $\nu_{i}$, which depended on how electrons were coupled, i.e. on how the "system" was structured as a whole and gave rise to the proper frequencies. ${ }^{25} \nu_{i}$ did not correspond to the resonant frequencies of individual electrons anymore, but to the resonant frequencies of the whole "system" of coupled electrons. The summation was always convergent. ${ }^{26}$

If we identify $4 \pi N^{\prime} M_{i}$ with $K_{i}$, Sellmeier's dispersion formula (formula (1)) and Schaefer's (formula (6)) are the same. Only the interpretation of $K_{i}$ changes. For Schaefer, $K_{i}=4 \pi N^{\prime} M_{i}$. According to Drude, instead, $K_{i}=4 \pi N_{i} e^{2} / m$ (formula (3)). Thus Schaefer's expression for $K_{i}$ was not proportional to the number of dispersion electrons $N_{i}$ resonating at $\nu_{i}$, as Drude's was, but to the number of systems $N^{\prime}$ and to still unknown properties of matter represented by $M_{i}$. Thus using Schaefer's expression it was impossible to count dispersion electrons from experiments, for $M_{i}$ could not be known a priori, like $e^{2} / \mathrm{m}$. Nothing could be said about dispersion electrons before understanding the structure of matter and how this was related to the parameter $M_{i}$. The notion of dispersion electrons lost its physical meaning. The new question was: what property of matter did $M_{i}$ exactly represent?

In the new framework, Drude's formula was reduced to a particular case of Schaefer's one, for the very simple case in which no coupling between electrons existed and resonant frequencies directly corresponded to the vibrations of individual electrons. But this was the least likely option. As Schaefer insightfully pointed out, if electrons were really independent from each other, no regularity in the ratios

\footnotetext{
${ }^{24}$ These solutions were called "Eigenfunctions" by David Hilbert (Hilbert, 1904a; Hilbert, 1904b). Each "Eigenfunction" had an "Eigenvalue" associated. Schaefer explicitly acknowledged Hilbert's papers and other mathematical papers about the theory of integral equations.

${ }^{25}$ Schaefer's original formulation is in terms of the light period $T$, instead of the frequency $\nu$. I have transformed Schaefer's equation for the sake of a better comparison between his and Drude's formulations. I have applied the conversion factor $T=\frac{2 \pi}{\nu}$. Nowadays it would be $T=\frac{1}{\nu}$, yet in textbooks and papers of that time, as for example Voigt's, physicists usually worked with a frequency $\nu$ that would nowadays be identified with the angular frequency. This frequency was related to the wavelength $\lambda$ in the following way: $\lambda=\frac{2 \pi c}{\nu}$, instead of $\lambda=\frac{c}{\nu}$, as it would be the case in current textbooks of physics.

${ }^{26}$ As a matter of fact, the summation had infinite terms, but it could be limited to $s$ terms without appreciable error.
} 
between different numbers of dispersion electrons $N_{i}$ resonating at different spectral lines would be expected. On the contrary, if regularities were detected, then electrons could not be independent from each other:

Würde man nun im Gegensatz dazu experimentell finden, z. B. dass $N_{1}=N_{2}=N_{3}=\ldots N_{s}$ ist, oder würde man finden, dass z. B. jedes folgende $N$ in bestimmter Weise aus dem vorhergehenden ableitbar (etwa im selben Verhältnis kleiner) ist, so würde man mit Recht schliessen, daß die verschiedenen Elektronengattungen, oder besser ausgedrückt, daß die verschiedenen Linien der Serie nicht unabhängig voneinander sein können (Schaefer, 1910, 884).

According to Schaefer, the independence hypothesis was "viel zu primitiv" and should be abandoned from the outset. Remarkably, Schaefer's claim did not undermine the dispersion formula, but only Drude's interpretation of it. The dispersion formula (formula (1)) remained structurally valid. The main difference between Drude's and Schaefer's formulas was the physical interpretation of the parameters of the numerator:

Man kann daher in gewissem Sinne sagen, dass der ganze Unterschied zwischen den beiden oben dargelegten Auffassungen auf die verschiedenen Interpretation der Produktfaktoren $N$ und $M$ herauskommt (Schaefer, 1910, 887).

Schaefer's critique had a positive side: one could use the calculations of the experimental values of $M_{h}$ as a way to explore the still unknown inner nature of atomic systems. In all, Schaefer's critique not only concerned the phenomenon of dispersion, but all theories of optical phenomena based on the same simple hypothesis that light interacted directly with the so-called dispersion electrons, such as the theory of the magnetorotation of light polarization, which according to Schaefer was nothing else than "eine erweiterte Dispersionstheorie" (Schaefer, 1909a, 432).

\subsection{From "dispersion electrons" to "systems": Ladenburg, 1912}

Schaefer's insights did not go unnoticed in Ladenburg's laboratory in Breslau. Ladenburg and Loria had experimentally observed what Schaefer mentioned in his 1910 paper as a theoretical possibility: regularities in the number of dispersion electrons vibrating at different spectral frequencies. More specifically, the systematic decrease of the number of dispersion electrons for increasing frequency of spectral lines observed by Ladenburg and Loria ever since 1909 hinted at the non-independence of electrons from each other. In 1912, Ladenburg resorted to Schaefer's considerations in order to interpret his findings (R. Ladenburg, 1912). This paper is a very good example of Ladenburg's high skills of both experimental performance and theoretical reflection.

Ladenburg's 1912 paper is unusually long and detailed in comparison to his previous papers on dispersion and magnetorotation of light polarization. There he reproduced his previous results on these two phenomena with improved precision (some systematic errors were eliminated) and incorporated new measurements for a comparative analysis (at different densities of gas and for different electric fields applied). In order to better explain his experimental innovations, he described at length the experimental setups and procedures. Further he complemented the experimental description with an insightful theoretical discussion about the number of dispersion electrons. Ladenburg used again monoatomic gas of hydrogen, despite the technical difficulties involved, because it offered better possibilities for a comparative 
analysis: it could be used at room temperature, the density could be easily controlled and the amount of electric field applied could be varied. Moreover, it played an important role in solar physics.

Ladenburg's 1912 main innovation was the systematic analysis of optical dispersion and magnetorotation of light polarization at different gas densities and for different electric fields applied (the amplitude of the electric field was theoretically proportional to the number of electrically excited atoms, and therefore to the number of atoms being optically active in the experiment). With these measurements Ladenburg went one step further with respect to his previous papers and the work of other experimentalists. These measurements gave Ladenburg a better understanding of the relationship between optical phenomena and the number of dispersion electrons. Interestingly, Ladenburg did not systematically adopt the language of "dispersion electrons," but he made extended use of another expression to denote the same thing: "centers of absorption". As a point of fact, on three occasions he identified the centers of absorption with dispersion electrons, yet throughout the text he systematically preferred the first expression, which was not associated to any specific particle. As expected, Ladenburg measured that, for increasing density and for increasing electric field, the value of the parameters $K_{i}$, assumed to be proportional to the number of absorption centers, always increased. In the first case, it was clearly a relation of proportionality. ${ }^{27}$ Nevertheless, the ratios of dispersion electrons vibrating at the different spectral lines, namely, $N_{H_{\alpha}} / N_{H_{\beta}}$, remained relatively constant for all variations of the gas density or electric field. Thus these ratios did not depend on external conditions, but on some aspect of the internal atomic or molecular structure.

In addition to these results, Ladenburg calculated again the number of dispersion electrons (or absorption centers) from the new improved experiments. He reached the same conclusions as in previous papers concerning the low number of dispersion electrons and, most importantly, the decrease of dispersion electrons for increasing frequency of the spectral line. In particular, he found only 1 electron per 10000 molecules resonating at the frequency of the $H_{\alpha}$ line. Further the ratio of dispersion electrons resonating at $H_{\beta}$ to the number of dispersion electrons resonating at $H_{\alpha}$ was found to be $N_{H_{\alpha}}=4,5 N_{H_{\beta}}$. Although it was impossible to measure the anomalous dispersion around $H_{\gamma}$ numerically, it was indisputable that in any case it was much smaller. Thus a systematic and fast decrease in the number of dispersion electrons per increasing spectral frequency, also perceptible in the experiments of (Wood, 1904), (Loria, 1909), and (Bevan, 1910) was corroborated once more.

This regularity was one of the most challenging results from an interpretative point of view. It could not be explained on the basis of usual theories of dispersion. Instead, Schaefer's theory offered a good alternative to understand these features. Ladenburg adopted Schaefer's term "system" to refer to the groups of electrons structured in atoms or molecules and he acknowledged that, according to Schaefer's theory, the various lines of the spectrum did not result from the vibration of individual electrons, but from the vibration of "systems". As a consequence, optical parameters were not proportional to dispersion electrons, but to still unknown properties of atoms and molecules in their way to interact, as a whole, with light. Since the value of these properties could be not be calculated a priori, as in Drude's theory, the calculations of the number of dispersion electrons made by assuming $K_{i}=4 \pi N_{i} \frac{e^{2}}{m}$ were not valid:

Dadurch bleibt zwar unsere Grösse $K$ der Zahl $N$ der "Systeme" proportional, (...) der Proportionalitätsfaktor ist jedoch nicht mehr konstant $4 \pi e^{2} / m$, sondern verschieden für verschiedene Serienglider; die Zahl $N$ der Systeme ist andrerseits für alle Glieder dieselbe und ist nicht ohne

\footnotetext{
${ }^{27}$ In the last part of the paper, Ladenburg used these experimental results to discuss the increasing width of spectral lines for increasing electric field and increasing gas density, as a result of the increasing number of absorption centers.
} 
weiteres aus Dispersionsversuchen zu entnehmen, so daß unsere oben berechneten absoluten Werte der Zahl der Resonatoren pro Volumenheit und die daraus gezogenen Folgerungen nicht gültig bleiben würden (R. Ladenburg, 1912, 301).

Ladenburg's conclusion concerned optical dispersion, as well as the magnetorotation of light polarization. As a matter of fact, Ladenburg's conclusion was not definitive, but tentative. Ladenburg had not fully abandoned Drude's theory in the paper, but he rather showed his openness to other theoretical alternatives. Most importantly, through all attempts to provide a good interpretation of experimental results, he pointed at one of the deepest flaws of the old optical theories: the presumed independence of electrons resonating with light.

In short, from 1908 to 1912, Ladenburg's way of dealing with optical dispersion had evolved in an interesting way: he began with an experimental challenge, then he used the experimental results to explore the microscopic structure of matter according to the current optical theories, and eventually he used his results to put into doubt the whole interpretation of optical theories in terms of dispersion electrons, holding on to the fact that light and matter interacted through resonance. The dispersion formula and other formulas of optical phenomena remained structurally the same. The whole issue was interpretive: resonance should no longer take place between light and electrons, but between light and atomic or molecular systems.

\subsection{The problem of dispersion electrons in a wider context: Ladenburg, 1914}

Two years later Ladenburg proceeded in more depth to the analysis of the concept of dispersion electrons and its flaws in a paper he published in 1914 (Ladenburg, 1914). This time he did not make new experiments, but he used already existing experimental data of another phenomenon, the reversal of spectral lines, which was presumably also caused by resonating electrons. This was the first optical phenomenon he explored in his laboratory, in 1908, as told in Section 3.1. The major contribution of this paper is indeed theoretical. Ladenburg proposed a new way to count dispersion electrons from experiments, by availing himself of new conceptual resources borrowed from another area of physics: thermodynamics. He could do so because he treated radiation of incandescent metals as thermal radiation.

\subsubsection{Radiation emitted by incandescent metals and thermal radiation}

Ladenburg's idea to treat the radiation emitted by incandescent metals as thermal radiation would not have come to him by chance. The two physicists whose experiments on thermal radiation had been crucial for the corroboration of Planck's law of blackbody radiation in 1900, were working at the University of Breslau, close to Ladenburg's laboratory: Otto Lummer and Ernst Pringsheim. ${ }^{28}$ What is more, throughout 1913 Ladenburg had been helping Hedwig Kohn, PhD student of Lummer and Pringsheim, with her experiments on thermal radiation. ${ }^{29}$ In her dissertation, published one year later (Kohn, 1914), Kohn experimentally confirmed that radiation emitted by incandescent bodies, such as metallic vapors, could be treated as thermal radiation.

\footnotetext{
${ }^{28}$ The secondary literature on this episode is huge. See for example (Jungnickel, 1986, 259-268) and (Mehra, 1982, 39-44).

${ }^{29}$ See the last paragraph of the paper out of her dissertation, where she thanks Lummer, Pringsheim for having inspired her work, and Ladenburg for having helped her in the experimental methods (Kohn, 1914). The dissertation was completed in 1913.
} 
For the radiation emitted by incandescent metals to be deemed as thermal radiation, it had to be proved that this radiation complied with Kirchhoff's law of thermal radiation. Thermal radiation was also known as blackbody radiation, for it was the kind of radiation emitted by so-called black-bodies, namely, ideally opaque and nonreflective bodies, which absorb all radiant energy falling upon then, reach a stationary state of thermal equilibrium at a certain temperature, and then reemit that energy. According to Kirchhoff, in a situation of thermal equilibrium, the energy emitted by a black-body was the same as the energy absorbed by the same body, integrated over the whole spectrum of frequencies. Mathematically it would be: ${ }^{30}$

$$
\int_{0}^{\infty} \epsilon_{\nu} d \nu=\int_{0}^{\infty} \alpha_{\nu} B_{\nu} d \nu
$$

in which $\epsilon_{\nu}$ was the emission coefficient of the body, depending on the light frequency $\nu, \alpha_{\nu}$ was the absorption coefficient of the body, also depending on the frequency, and $B_{\nu}$ was a universal function that accounted for the intensity of polarized radiation coming from only one direction at a specific frequency $\nu$. Kirchhoff's law had the following implication:

$$
B_{\nu}=\frac{\epsilon_{\nu}}{\alpha_{\nu}}
$$

This formula meant that, inside a medium in thermal equilibrium, the radiation intensity at one frequency coincided with the quotient between the emission coefficient and the absorption coefficient at the same frequency. The form of the function $B_{\nu}$ did not depend on the nature of the medium, but it only depended on the temperature of the medium. The radiation intensity was related to the density of radiation coming from all directions in the space and having all possible polarizations, $u_{\nu}$, through the following expression:

$$
u_{\nu}=\frac{8 \pi B_{\nu}}{c}
$$

in which $c$ denoted the velocity of light.

In 1900, the prominent German physicist Max Planck theoretically derived a radiation law for $u_{\nu}$ that fitted perfectly the experimental results: ${ }^{31}$

$$
u_{\nu} d \nu=\frac{8 \pi h \nu^{3}}{c^{3}} \frac{1}{\left(e^{h \nu / k T}-1\right)} d \nu
$$

in which $h$ is a universal constant, called Planck's constant, and $K$ is Boltzmann's constant. Planck's radiation law represents the distribution of the energy emitted by a black-body over all frequencies of the spectrum.

Kohn's method to confirm that radiation emitted by incandescent metal vapors was nothing but thermal radiation implied the use of Planck's radiation law. On the one hand, Kohn measured the temperature of the incandescent lamp T. On the other hand, she measured experimentally the relation $\frac{\epsilon_{\nu}}{\alpha_{\nu}}$ at the spectral lines. Eventually, by means of Planck's radiation law, she computed from $\frac{\epsilon_{\nu}}{\alpha_{\nu}}$ the temperature $T_{1}$ that should correspond to the incandescent lamp when this emitted thermal radiation.

\footnotetext{
${ }^{30}$ See (Planck, 1906) for more details.

${ }^{31}$ There is a huge literature on Planck's radiation law. See for example: (Jammer, 1966), (Kuhn, 1978), and more recently (Badino, 2015).
} 
If one were to find that $T=T_{1}$ systematically, then the radiation emitted by the incandescent lamp could be regarded as thermal, which was the case. ${ }^{32}$

The consideration of the radiation emitted by incandescent metal vapors as thermal radiation had important consequences. If radiation was due to chemiluminescence, its characteristics would depend on the chemical structure of the gas. If instead radiation was thermal radiation the distribution of energy radiation over frequencies would follow Planck's universal radiation law and would not depend on the particular structure of each substance, but only on the temperature of the gas. Under these circumstances, Ladenburg could resort to Planck's conceptual resources, in particular, to the so-called Planck's resonators.

In his derivation of the radiation law, Planck had modeled a black-body as a set of electric resonators enclosed in a cavity with perfectly reflecting walls. Each resonator had only one frequency, and interacted only with the component of the electromagnetic field that had the same frequency. For Planck, such electromagnetic resonators were not real objects, but abstract objects symbolically represented by a mass, dipole moment and natural frequency, which allowed him to deal with the problem of a black-body interacting with electromagnetic light without having to hypothesize anything about the microstructure of matter. ${ }^{33}$ One of the key results of his theoretical research on this model was his famous relation between the average energy $U$ of a resonator of frequency $\nu$ and the spectral density of thermal radiation $u_{\nu}$ at the same frequency, when both were in thermal equilibrium:

$$
U=\frac{c^{3}}{8 \pi \nu^{2}} u_{\nu}
$$

This expression and Planck's resonators played a crucial role in Ladenburg's 1914 paper.

Famously, in order to derive the right expression for radiation density that fitted well to experimental data, Planck had to further introduce a novel hypothesis in 1900: his abstract resonators could not hold all possible energy values, but only a multiple of a discrete amount of energy $E=h \nu$, called quantum of energy. ${ }^{34}$ Nevertheless, in the 1914 paper Ladenburg did not use the discontinuity hypothesis. ${ }^{35}$

\subsubsection{Ladenburg's argument: dispersion electrons as Planck's resonators}

The gist of Ladenburg's argument was the following: the total amount of energy emitted at one spectral line by an incandescent gas of known volume and temperature $T$ (treated as thermal radiation), divided by the average energy emitted per unit time by one Planck's resonator, was the number of "emission centers" of the sample. Indeed, the "emission centers" corresponded to dispersion electrons. Then, by using experimental data of the energy emitted by an incandescent gas at its spectral

\footnotetext{
${ }^{32}$ Obviously, such a methodology required the existence of a well-founded distribution law of radiation. Thus before 1900, this kind of experiments were rather problematic. But even after 1900, the results led to no consensus, for some measurements led to the conclusion that the chemical state of the gas, apart from the temperature, determined the amount of energy transmitted.

${ }^{33}$ See (Badino, 2015, 44-45).

${ }^{34}$ Literature on Planck's contribution is also huge. For a discussion of how revolutionary Planck's ideas were, see for example (Darrigol, 2001), (Gearhart, 2002), (Badino, 2009), and (Badino, 2015).

${ }^{35}$ Ladenburg was well informed about quantum theory. In 1909 he even wrote a daring and wellinformed paper about the photoelectric effect and X-Ray phenomena, in which he clearly favored Einstein's idea of lightquanta (Ladenburg, 1909b). Yet he explicitly mentioned that, although emission presumably took place in the form of quanta, the average emission and absorption energies were not affected and evolved continuously. The discontinuity assumption was not necessary in order to use the expression (11).
} 
lines, it was possible to calculate the number of dispersion electrons emitting at that frequency. Such an argument implied that Planck's abstract resonators, used as a heuristic tool to analyze the properties of black-body radiation, were now identified with real physical entities.

Ladenburg's argument proceeded along the following steps.

First of all, Ladenburg followed closely Planck's 1906 textbook Vorlesungen über die Theorie der Wärmestrahlung (Planck, 1906) to compute the average energy emitted per unit time by one emission center (Planck's resonator), which he identified with an electron of charge $e$ and mass $m .{ }^{36}$ In particular, Ladenburg arrived at the following expression:

$$
H=\frac{8 \pi^{2} e^{2}}{m c} B(\nu)=\frac{\pi e^{2}}{m} u_{\nu}
$$

in which $B(\nu)$ was the intensity of a polarized beam of frequency $\nu$, and $u_{\nu}$ the spectral density of thermal radiation when all possible directions and polarizations were taken into account.

Secondly, Ladenburg related the total amount of emitted energy at one spectral frequency with the number $N$ of emission centers resonating at the same frequency. To do so he drew upon Kohn's above-mentioned work in which she proved that incandescent radiation emitted by metallic vapors could be treated as thermal radiation. Therefore Ladenburg could use the typical expression $B_{\nu}=\frac{\epsilon_{\nu}}{\alpha_{\nu}}$ for thermal radiation to characterize the energy emitted and absorbed by an incandescent metallic gas. Then he assumed that the total energy emitted per unit time by a unit volume of an incandescent metallic gas, which was proportional to the total energy absorbed per unit time by the same gas, was $H$ multiplied by $N$ emission centers:

$$
8 \pi \int_{0}^{\infty} \epsilon_{\nu} d \nu=8 \pi B(\nu) \int_{0}^{\infty} \alpha_{\nu} d \nu=N H
$$

The integral had a significant value only within a very narrow interval around the spectral line. $\epsilon_{\nu}$ and $\alpha_{\nu}$ were the emission and absorption coefficients of a black-body, respectively. As a consequence:

$$
\int_{0}^{\infty} \alpha_{\nu} d \nu=\frac{\pi N e^{2}}{m c}
$$

Actually, to prove the universal character of the above-expression, Ladenburg calculated the above-integral for different functions of $\alpha_{\nu}$ corresponding to different forms of damping (as for example Doppler effect or some kind of frictional forces) and always arrived at the same relationship between $\int_{0}^{\infty} \alpha_{\nu} d \nu$ and $N$.

Thirdly, Ladenburg related the above-mentioned results to quantities that could be directly calculated from experiments. To do so, he did not resort to any optical theory based on specific mechanisms of light-matter interactions, but two empirical laws suggested in the 18th century, which related the radiating intensity to the direction of emission (Lambert's cosine law) and to the thickness of the emitting body (Biot-Lambert law). More specifically, Ladenburg made use of two papers recently

\footnotetext{
${ }^{36}$ This average amount of emitted energy $H$ was not the same as the average energy $U$ of a resonator, though both quantities were related through the expression $H=6 \sigma \nu U$, in which $\sigma$ was the damping constant of resonators. The energy lost and gained by Planck's energy from thermal radiation was characterized by the damping constant. In order to interact with the surrounding radiation, Planck's resonators could obviously not conserve their energy.
} 
written by himself and two Breslau colleagues in which these two empirical laws were applied and further developed.

The first paper was a theoretical-experimental paper written in 1910 by Lummer and Reiche (Lummer and Reiche, 1910), in which they mathematically extended Lambert's cosine law to the case in which the radiating material had a finite thickness. Lummer and Reiche also proved their conclusions experimentally. The general law established that the radiant intensity emitted by an ideal radiator in one specific direction was directly proportional to the cosine of the angle between the direction of the incident light and the normal direction to the radiator surface $(\theta)$. Integrating over the whole space, one obtained the total energy emitted by the radiating source in all directions, at an specific frequency. In case that the emitting source had a finite thickness $D$, the law was slightly modified to include the new parameter. According to Lummer and Reiche, as reproduced in Ladenburg's 1914 paper, the energy $E$ emitted by a source of thickness $D$ in a direction that formed an angle $\theta$ with the normal direction of the emitting surface was:

$$
E=\frac{2 \epsilon_{\nu} \cos \theta}{\alpha_{\nu}}\left(1-e^{\frac{\alpha_{\nu} D}{\cos \theta}}\right) d \Omega
$$

in which $\epsilon_{\nu}$ and $\alpha_{\nu}$ were the emission and absorption coefficients of the radiating source, respectively, and $d \Omega$ was the solid angle of the elementary surface that received the radiation from a source at a distance $r$, in a direction that formed an angle $\theta$ with respect to normal direction the emitting surface. If $D$ was infinite (ideal case), the law was reduced to Lambert's law.

Ladenburg transformed the above expression by assuming very small values of $\frac{\alpha_{\nu} D}{\cos \theta}$, that radiation was thermal radiation, namely, $B_{\nu}=\frac{\epsilon_{\nu}}{\alpha_{\nu}}$, and that all frequencies from 0 to $\infty$ were contributing. Further, by making use of the expression (13), he obtained the following expression:

$$
E=\frac{N H D}{4 \pi} d \Omega\left(1-\frac{D \int_{0}^{\infty} \alpha_{\nu}^{2} d \nu}{2 \cos \theta \int_{0}^{\infty} \alpha_{\nu} d \nu}\right)
$$

This expression related the energy received at some point of the space coming from a source (black-body) emitting at all frequencies to the number $N$ of emitting centers (or dispersion electrons) of the source.

Ladenburg could continue transforming this formula by taking into account the second paper mentioned above, written by himself and Reiche in 1913 (Ladenburg and Reiche, 1913). It was a theoretical paper based on the so-called Biot-Lambert law (today better known as Lambert or Beer-Lambert law). According to this law, the intensity of light transmitted across an absorbent medium $J_{t}(\nu)$ was related to the intensity of light that initially fell upon the sample $J_{0}(\nu)$ through the following expression:

$$
J_{t}(\nu) d \nu=J_{0}(\nu) d \nu e^{-2 \alpha_{\nu} D}
$$

in which $\alpha_{\nu}$ was the absorption coefficient of the absorbing material and $D$ was the path length of light through the sample.

In 1913 Ladenburg and Reiche applied this law to the special situation in which a body simultaneously radiated and absorbed light at spectral frequencies. This situation is the same that is reproduced in experiments of reversal of spectral lines, which Ladenburg had already explored in 1908 with monoatomic gas of hydrogen 
simultaneously emitting and absorbing at the spectral lines (Ladenburg, 1908). More specifically, what Ladenburg and Reiche calculated in 1913 was the total amount of energy absorbed in this case, what they called "line absorption" $\left(A_{D}\right)$, given the body had a thickness $D$.

What Ladenburg realized in 1914 is that $\frac{D \int_{0}^{\infty} \alpha_{\nu}^{2} d \nu}{\int_{0}^{\infty} \alpha_{\nu} d \nu}$ corresponded exactly to what he and Reiche had called "line absorption" in their 1913 paper, which was experimentally measurable. After substituting $\frac{D \int_{0}^{\infty} \alpha_{\nu}^{2} d \nu}{\int_{0}^{\infty} \alpha_{\nu} d \nu} 37$ by $A_{D}$ and having solved the integrals for $d \nu$ and for $d \Omega$ (given an aperture angle $2 \theta_{1}$ of the collimator of the experimental setup), Ladenburg obtained a more simplified expression for the energy received at the collimator of the experimental source by a source (black-body) of thickness $D$ emitting (and simultaneously absorbing) at the spectral lines. This expression continued depending on the number $N$ of "emission centers" of the body that presumably resonated at the frequencies of the spectral lines:

$$
E=\frac{N H D}{2}\left(1-\cos \theta_{1}\right)\left(1-\frac{A_{D}}{2}\right)
$$

Eventually, the above expression could be directly related to another experimentally measurable parameter, the so-called luminance $i$, instead of being referred to $E$. The luminance was defined as the ratio between the real energy emitted by radiating bodies at spectral lines that simultaneously absorbed energy at the same frequencies to the total radiating energy emitted by an ideal black-body in the same direction and at the same frequency $\left(E=2 \pi B(\nu) \Delta \nu \sin ^{2} \theta_{1}\right) .{ }^{38}$ If $\theta_{1}$ was considered to be very small, the luminance $i$ turned out to be related to the number $N$ of emission centers in the following way:

$$
N D=\frac{8 \pi i \triangle \nu}{C\left(1-\frac{A_{D}}{2}\right)}
$$

in which $D$ was the path length of the gas and $C$ was $C=\frac{8 \pi^{2} e^{2}}{m c}$. Both $\triangle \nu$ and $A_{D}$ were also variables whose value could be computed from experiments, like $i$. $\triangle \nu$ was the small range of frequencies over which the spectral line stretched out. $A_{D}$ was the "line absorption".

Thus Ladenburg had obtained another expression that related the number $N$ of emission centers (identified with dispersion electrons or "emitting electrons") to measurable parameters in optical experiments, without assuming any specific mechanism of absorption and emission of light. He only assumed that radiation emitted by incandescent bodies at spectral lines could be identified with thermal radiation, which allowed him to resort to Planck's resonators instead of Drude's and Voigt's electron theories in optics. The luminance could be measured in experiments of reversal of spectral lines.

\footnotetext{
${ }^{37}$ The integral had a significant value only at the spectral lines.

${ }^{38}$ For a derivation of this formula, see (Planck, 1906).
} 


\subsubsection{Counting dispersion electrons from experiments of reversal of spectral lines}

To use the expression (19) for computing numbers of dispersion electrons, Ladenburg did not rely on measurements of dispersion or magnetooptical experiments, but on measurements of the reversal of spectral lines by incandescent metallic vapors. More specifically, Ladenburg made use of existing data for sodium vapor at the spectral lines $D_{1}$ and $D_{2}$ (Gouy, 1879). Nevertheless, Ladenburg reached the same conclusions as with experiments of dispersion and magnetorotation of light polarization: a very low proportion of electrons per atom were participating (between 13 and 27 per cent of atoms involved), and the number of dispersion electrons decreased from $D_{2}$ to $D_{1}$, more specifically, $N_{D_{2}} / N_{D_{1}}=2$.

Despite some disagreements concerning the empirical value of the ratio $N_{D_{2}} / N_{D_{1}}{ }^{39}$ the most crucial aspect was that all phenomena led to the same regularity about the number of dispersion electrons: the low proportion of electrons and the decrease of its number with frequency of the spectral line. Ladenburg hinted again at the possibility of coupling between electrons inside the atoms. In addition to Schaefer's papers, Ladenburg quoted another paper about electronic coupling written by Voigt (Voigt, 1913). To explain the Zeeman effect, Voigt had suggested that electrons were coupled in pairs, so that the displacement of one electron under the action of an external magnetic field depended on the displacement of the other. In this case, optical equations referred to the number $N$ of coupled electronic pairs, instead of the number $N$ of individual resonating electrons:

Was die theoretische Seite unserer ganzen Fragestellung betrifft, so würde die Annahme, dass ein Atom zugleich mehrere Spektrallinien emittiert, d. h., dass die betreffenden Elektronen ein gekoppeltes System bilden, natürlich zu einer anderen als Gleichung 1 ) fuhren. ${ }^{40}$ So hat Voigt bekanntlich zur Erklärung der komplizierten Zeemaneffekte mit grossen Erfolge die Hypothese durchgeführt, dass z. B. die beiden D-Linien gekoppelt sind, und hat aus verschiedenen Verhalten der beiden Linien geschlossen, dass die Zahl der Freiheitsgrade, auf denen die $D_{2}$-Linie beruht, doppelt so gross ist wie diejenige von $D_{1}$. Diesem Schluss würden die hier gezogenen Folgerungen natürlich nicht widersprechen (Ladenburg, 1914, 777).

Obviously, Voigt's considerations on the different number of degrees of freedom corresponding to $D_{2}$ and $D_{1}$ nicely fitted with Ladenburg's calculations of the ratio $N_{D_{2}} / N_{D_{1}}=2$ from experiments. If electrons formed coupled systems, $N$ could not refer to the number of individually resonating electrons, thus it lost its original meaning. The question remained open: what was the meaning of $N$ ?

In summary, Ladenburg had reached the same conclusions in 1914 as in 1912, yet following a completely innovative method: he applied the notion of dispersion electrons (in this case emitting electrons) to another phenomenon (intensity of spectral lines) and, most importantly, availed himself of radically new conceptual resources from a different area in physics: thermodynamics of black-body radiation. In doing so Ladenburg identified the abstract and symbolic resonators of Planck's theory to hypothetically real physical entities that could be directly characterized from experiments, while avoiding any hypothesis on the microstructure mechanism of light-matter interaction.

\footnotetext{
${ }^{39}$ In fact, this was also the relation that Dimitri Roschdestwensky had obtained in 1912 by means of a rather innovative method to measure dispersion (Roschdestwensky, 1912). Loria had obtained 1,3 .

${ }^{40}$ The equation (1) was the one relating the energy $H$ emitted by one emitting center to the values $e$ and $m$ of one electron, namely, $H=\frac{8 \pi^{2} e^{2}}{m c} B(\nu)$.
} 
It was not until 1921 that Ladenburg returned to the issue. The reason was the Great War. During this time, the new quantum model of atom put forward in 1913 by the young Danish physicist Niels Bohr had made its way through. Rather than being an obstacle, in 1921 Ladenburg used Bohr's atomic model to answer the question he posed in 1914, while keeping the core of his 1914 argument untouched: that the total amount of energy emitted at one spectral line was proportional to the number of Planck's resonators, these being identified with electrons. Unfortunately, Ladenburg's 1914 contribution went unnoticed. From 1914 to 1921 other physicists attempted to explain optical dispersion in the context of Bohr's atomic model by ignoring experiments of dispersion around the spectral lines and their related interpretative problems.

\section{Bohr's atomic model during the WWI}

\subsection{Bohr's atomic model and dispersion between 1913 and 1915}

In 1913, Niels Bohr published a trilogy in which he put forward the first quantum model of atom (Bohr, 1913a; Bohr, 1913b; Bohr, 1913c). ${ }^{41}$ According to it, light and matter exchanged energy in a radically different way from the process of resonance described by optical theories. Bohr deemed atoms as microscopic planetary systems, and postulated that within them electrons moved around the nucleus in stationary orbits, from which no energy was radiated. From the point of view of ordinary electrodynamics, this was impossible. From the point of view of ordinary electrodynamics, rotating electrons gradually lost energy while being accelerated, thus an orbit could not be simply stationary.

But still more unconceivable was his second postulate: the emission and absorption of light by atoms was caused by instantaneous jumps of electrons between two different stationary states. According to Bohr, the frequency $\nu$ of emitted/absorbed light did not coincide with the frequency of any mechanical motion of particles, but was determined by the difference of energies between the initial and final stationary states involved in the quantum jump, through the expression $E_{1}-E_{2}=h \nu, h$ being the famous Planck's constant. The process meant a radical break with the model of resonance, according to which matter absorbed and emitted energy as continuous and mechanical processes when both matter vibrations and light waves were in tune. Now, according to Bohr's atomic theory, light frequencies did not coincide any longer with the frequency of mechanical vibration of microscopic particles, but were related to a non-mechanical and non-electrodynamic process, namely, quantum jumps. This was the first time that the quantum discontinuity became relevant to explain the inner structure of matter and its interaction with light. ${ }^{42}$

Arnold Sommerfeld, Peter Debye and Max Born became the first adepts to Bohr's theory in Germany, after Bohr had traveled to Germany in 1914 and had given several talks in Munich and Göttingen. Arnold Sommerfeld was at that time perhaps the most prominent German theoretical physicist (Eckert, 2013). Since 1906 he was full professor of physics and director of the new Theoretical Physics Institute at the University of Munich. Peter Debye had been Sommerfeld's first assistant in Munich, and since 1913 was at the University of Göttingen. Max Born was at the University of Göttingen at that time as well, where he remained until 1915, when he moved

\footnotetext{
${ }^{41}$ The full section is a summary of (Jordi Taltavull, 2013a) and chapter 4 of (Jordi Taltavull, 2019).

${ }^{42}$ For a deeper analysis of Bohr's trilogy, see (Heilbron, 1969), (Rosenfeld, 1981), (Kragh, 2012), and (Aaserud, 2013), among others.
} 


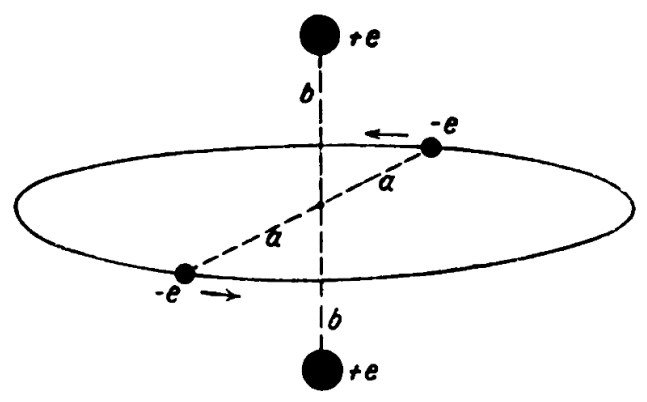

Fig. 9. Reproduction of Bohr's model for the $H_{2}$ molecule in Fritz Reiche's 1921 textbook of the quantum theory (Reiche, 1921, 98).

to Berlin. The Great War had already started and Born joined Ladenburg in the Artillerie Prüfungs-Kommission for artillery research (Born, 1975). ${ }^{43}$

Given the paradigmatic role of optical dispersion in explaining magnetooptical phenomena and the apparent conceptual incompatibility between the model of resonance and quantum jumps, it is not surprising that, early after becoming adepts of Bohr's atomic model, Debye and Sommerfeld tried to explain optical dispersion in the new atomic context. In fact, Debye's and Sommerfeld's first contributions to Bohr's atomic model, appeared in 1915, were about optical dispersion. Bohr's major achievement had been to explain the generation of spectral lines of atomic hydrogen (Balmer series). Nevertheless, most atomic and molecular spectra as well as optical dispersion remained unexplained by Bohr's atomic model. Spectral lines and dispersion differed in an essential aspect: spectral lines amounted to practically monochromatic radiation, which could be identified with quantum frequencies. Optical dispersion, instead, consisted of the continuous spread of light over the whole range of frequencies, only interrupted at certain singular frequencies, at which light was absorbed, and not dispersed. It was not a discontinuous, but a continuous phenomenon, which could not simply be explained by means of instantaneous quantum jumps.

The basic idea of Debye and Sommerfeld was to reconcile quantum postulates with ordinary electrodynamics by making quantum jumps and resonance coexist within atoms and molecules. Peter Debye developed in 1915 the first full-fledged theory of optical dispersion on the assumption that electrons could both mechanically vibrate around their stationary states and also perform quantum jumps to change their stationary state (Debye, 1915). ${ }^{44}$ Debye developed his theory for the case of hydrogen molecule $\mathrm{H}_{2}$, which consisted of two hydrogen atoms. He chose a model of molecular hydrogen that was already figured out by Bohr in his second installment of the trilogy: two hydrogen nuclei, whose symmetry axis was orbited by two electrons on a single ring (see Fig. 9).

\footnotetext{
${ }^{43}$ To be sure, in the beginning Bohr's atomic model was not well received in Continental Europe, in particular, in Germany. The early responses to Bohr's atom came mostly from British physicists. This is not surprising, for Bohr stayed in England for most of the period 1912-1915 and at the beginning he published in British journals, like Nature and Philosophical Magazine (Kragh, 2012, 100-116). The apparent "free will" of electrons in performing quantum jumps and the non-radiative nature of stationary states were two key aspects that provoked either rejection or skepticism among the community of physicists (Kragh, 2012, 90-139).

${ }^{44}$ In fact, in 1913 Bohr had already proposed that electrons were not just able to perform quantum jumps, but also to vibrate mechanically about their stationary orbits, in a perpendicular direction to the orbital plane (Bohr, 1913b). This second kind of continuous motion should be responsible for optical dispersion. Yet, as always, Bohr was rather ambiguous in developing these ideas and in discussing them in relation to experiments on spectral lines and dispersion.
} 
More specifically, he carried out a perturbation analysis of the orbits under the influence of electromagnetic waves. He allowed 5 possible electronic vibrations around the stationary state. Each possible vibration was characterized by a proper frequency, represented by a singularity in the dispersion formula. In this way, dispersion continued to be explained on the basis of an ordinary process of resonance between electromagnetic light and vibrating electrons. If light frequency coincided with some of the 5 proper frequencies of electronic vibration around their orbits, matter and light were set into resonance. For the other frequencies, light was transmitted and delayed. The basic difference with Drude's optical theory was that vibrations did no longer take place around equilibrium positions, but around stationary states.

The comparison between the theoretical values of resonant frequencies and experimental data with $\mathrm{H}_{2}$ turned out to be quite satisfactory. Debye compared his theoretically derived formula with empirical data obtained by the experimental physicist John Koch in 1913 (Koch, 1913). Koch measured optical dispersion through a gas of $\mathrm{H}_{2}$. No absorption frequencies could be directly observed, since all of them lay far away from the visible range of frequencies. Nevertheless, Koch was able to compute their value by fitting his own experimental data into a common dispersion formula with singularities at the absorption frequencies. ${ }^{45}$

Given Debye's success, Arnold Sommerfeld took up Debye's approach and generalized it to all types of molecules with axial symmetry (Sommerfeld, 1915). As in the case of Debye, Sommerfeld's treatment relied on the mechanical perturbation induced to stationary orbits by electromagnetic radiation. Eventually, he obtained a generalized version of Debye's formula for the index of refraction as a function of the frequency of light:

$$
n^{2}-1=\frac{4 \pi e^{2}}{m} \sum_{i=1}^{\infty} \frac{N \mu \frac{C_{i}}{3}}{s_{i}^{2}-\nu^{2}} .
$$

Here $\mu$ is the number of electrons per molecule, $N$ the number of molecules per unit volume, $s_{i}$ the proper frequencies at which orbiting electrons were supposed to resonate with light, and $C_{i}$ a constant that accounts for the anisotropy of the material. $s_{i}$ are indeed proportional to the angular velocity $\omega$ of the ring via certain factors $x_{i}$, namely, $s_{i}=\omega x_{i}$, but they do not coincide with angular frequencies. The resonance frequencies $s_{i}$ of the system coincide with the vibration frequencies of electrons around their orbits. The summation extends over all possible resonance frequencies of the system.

Sommerfeld's formula was again analogous to Drude's, yet also with some interpretive differences. ${ }^{46}$ Though both formulas were structurally the same and presented singularities at resonance frequencies, according to Sommerfeld electronic vibrations did no longer take place around equilibrium positions, but around stationary states. Moreover, though both formulas were proportional to the number of dispersion electrons $N \mu$ vibrating at those resonance frequencies, now $N \mu$ was weighted by a factor $\frac{C_{i}}{3}$, which was due to the anisotropy of the molecule.

In all, Sommerfeld had succeeded in developing a theory of optical dispersion that continued to be essentially classical, based on the old mechanism of resonance, yet within a quantum structure of matter. ${ }^{47}$ Therefore Sommerfeld was very optimistic

\footnotetext{
${ }^{45}$ Hence dispersion experiments using $\mathrm{H}_{2}$ were different from dispersion experiments with solar gases in a crucial aspect: $\mathrm{H}_{2}$ spectrum was continuous at the visible range of frequencies (absorption frequencies could only be calculated a posteriori by extrapolating experimental data), whereas the spectrum of solar gases had visible discontinuities at spectral lines.

${ }^{46}$ Insert Drude's expression for $K_{i}$ (Eq. (3)) into Sellmeier's dispersion formula (Eq. (1)) in order to compare Drude's and Sommerfeld's dispersion formulas.

${ }^{47}$ To be sure, the comparison between experiments and theory only worked out for the molecule of $H_{2}$. Sommerfeld imagined theoretical models for $\mathrm{N}_{2}$ and $\mathrm{O}_{2}$ in which all electrons were equidistant
} 
about the coexistence between classical $^{48}$ mechanics and electrodynamics, on the one hand, and quantum jumps, on the other hand. Such a coexistence relied on a sharp "division of labour" between classical physics and quantum physics: quantum physics accounted for essentially discontinuous phenomena, such as spectral lines, while classical physics remained valid to account for continuous optical phenomena, such as optical dispersion.

Yet not all that glitters is gold. Sommerfeld's dispersion theory and strong separation between classical and quantum domains had important limitations which were acknowledged by Sommerfeld himself. The fact is that at exactly the proper frequencies $s_{i}$, where the dispersion was discontinuous, Sommerfeld's theory collapsed. Two possible explanations were possible at the same time. On the one hand, according to the model of resonance, $s_{i}$ were considered the proper frequencies at which light was absorbed during dispersion by resonance. On the other hand, according to Bohr's theory, those absorption frequencies should coincide with the emission (and thus spectral) frequencies, at which the exchange of electron energy with light was governed by quantum jumps. Sommerfeld's way out of the conflict was to state that his dispersion theory was valid only far away from emission and absorption frequencies, that is to say, just for normal dispersion. This was the case for $\mathrm{H}_{2}$, whose spectrum was continuous over the whole visible range of frequencies. About anomalous dispersion, that is, dispersion in the neighborhood of absorption frequencies, he could say nothing. In his own words:

Wie man die anomale Dispersion und die hierbei erforderliche Elektronendämpfung anzusetzen hat, liegt noch in tiefem Dunkel (Sommerfeld, $1915,577)$.

Anomalous dispersion was precisely what Wood's, Bevan's, and Ladenburg's experiments were about. Either because Sommerfeld did not know about them or because they fell out the range of validity of his theory, Sommerfeld did not ever mention this kind of experiments. As if they did not exist.

\subsection{The flaws of Debye-Sommerfeld theory}

Within a few years the Debye-Sommerfeld dispersion theory collapsed, despite some physicists initially embraced it. ${ }^{49}$ Different kinds of arguments were used against the Debye-Sommerfeld theory, but the most relevant became the experiments of dispersion around the spectral lines. ${ }^{50}$ It was Bohr who first raised anomalous dispersion as one of the strongest objections against Debye and Sommerfeld's theory. ${ }^{51}$ According

\footnotetext{
from each other and orbited the nucleus in only one ring. Given this specific geometrical distribution, he calculated the theoretical values of the constants of the dispersion formulas. The problem was that the theoretical values did not coincide with the value of the constants obtained from experimental data. Yet Sommerfeld did not see this disagreement as a reason to quit his theory. He rather thought that the specific molecular models for $\mathrm{N}_{2}$ and $\mathrm{O}_{2}$ should be much more complicated than just one ring for all molecular electrons.

${ }^{48}$ This is exactly the word Sommerfeld used: classical mechanics and electrodynamics, as the opposite of quantum physics.

${ }^{49}$ For example, Adalbert Rubinowicz extended it to the vibration of nucleus (Rubinowicz, 1917). See also (Epstein, 1916).

${ }^{50}$ Other arguments than dispersion at spectral lines were provided by the Dutch physicist Hendrika Johanna van Leuween, on the one side, and Carl Wilhelm Oseen, on the other side. van Leuween argued that mechanical oscillations of $\mathrm{H}_{2}$ would render the molecule unstable (Leeuwen, 1916). Oseen argued that the electromagnetic theory and Bohr's atomic model were simply conceptually incompatible (Oseen, 1915). For further discussion, see (Jordi Taltavull, 2013a) and (Jordi Taltavull, 2019).

${ }^{51}$ See also (Epstein, 1922).
} 
to him, if experiments of anomalous dispersion showed that absorption frequencies coincided with spectral lines in the visible range of frequencies, one should expect the same coincidence for absorption frequencies falling outside the visible range. If this was the case, dispersion frequencies could not be accounted for by means of ordinary electrodynamics. In Bohr's own words, extracted from a letter sent to Oseen, on December 20th 1915:

It appears, e.g., from Wood's and Bevan's experiments on the dispersion in sodium and potassium vapors that the characteristic frequencies which determine the dispersion coincide with the frequencies of the principal series in the Sodium and Potassium spectra, and one must therefore expect that the same thing holds for other gases, e.g. helium, only that the corresponding line lies far out in the ultraviolet. If these frequencies are determined by the laws for quantum emission, the dispersion cannot, whatever its explanation, be calculate from the motion of the electrons and the usual electrodynamics, which does not have the slightest connection with the frequencies considered (Hoyer, 1981, 337-338).

One year later, Bohr went still further. On the basis of Wood's and Bevan's experimental results, he argued that any dispersion theory had to depend on the mechanism of transitions between different stationary states. The problem was to figure out how this connection was possible. Since instantaneous jumps could not account for the continuous dependency of the index of refraction on the frequency of light, Bohr put forward an analogy with ordinary electrodynamics to describe a possible mechanism causing dispersion:

If the above view is correct and the dispersion depends on the mechanism of transition between different stationary states, we must, on the other hand, assume that this mechanism shows a close analogy to an ordinary electrodynamic vibrator (Hoyer, 1981, 449).

Furthermore, based on the empirical asymmetry in the number of electrons vibrating at different spectral lines, Bohr suggested that the change of number of electrons from one proper frequency to another was somehow connected with the "greater tendency" of the quantum jumps to occur between successive stationary states than between more distant states.

To be sure, Bohr did not go beyond a simple analogy. Moreover, Bohr's 1916 paper was eventually withdrawn. ${ }^{52}$ Nevertheless, it became clear that experiments of anomalous dispersion around spectral lines could not be simply ignored. Bohr wrote about them to Sommerfeld in a letter sent in 1916 (Hoyer, 1981, 604). Sommerfeld continued resisting to the idea of a quantum mechanism explaining dispersion (Sommerfeld, 1917), yet in 1920 he eventually gave in. For one, the model of $H_{2}$ with axial symmetry, which offered the most important experimental support to his dispersion theory, was troubled with many difficulties. ${ }^{53}$ Also, Sommerfeld regarded Wood's experiments of anomalous dispersion around sodium vapor as "besonders entmutigend", for they were an evidence that the dispersion characteristic frequencies, coinciding with the spectral lines, also required a quantum theoretical explanation (Sauter, 1968, 500). The problem was that nobody knew which explanation would be probable.

\footnotetext{
${ }^{52}$ Bohr decided to postpone its publication in the light of another contribution published by Sommerfeld in the same year, in which Sommerfeld famously extended Bohr's quantization to noncircular orbits (Sommerfeld, 1916a; Sommerfeld, 1916b). The two papers are reproduced in (Sauter, 1968). There is a huge literature on it. See for example: (Heilbron, 1967, 466-470), (Nisio, 1973), (Kragh, 1985), (Seth, 2010, 162-171), (Eckert, 2000, 436-441), (Eckert, 2013, 268-279).

${ }^{53}$ In particular, in the areas of specific heats, magnetic properties, dissociation heat, band-spectra. See (Jordi Taltavull, 2013a) and chapter 4 of (Jordi Taltavull, 2019).
} 


\section{Reinterpreting “dispersion electrons” after WWI}

\subsection{Ladenburg and Bohr's atom}

It was Ladenburg, in 1921, who took the first steps towards a radical reinterpretation of optical dispersion on the basis of Bohr's atom. WWI was the reason why Ladenburg did not get back to the dispersion problem until 1921. In the beginning, he served as official of cavalry in the field, but then he convinced the military authorities of the Artillerie-Prüfungs-Komission to organize a department for sound ranging in Berlin, and he was in charge of it until the end of the war (Born, 1975). It was not until he came back to Breslau in 1919 that he again started publishing scientific papers. By then he was perfectly aware of Bohr's atomic theory and had fully embraced it.

Ladenburg must have known of Bohr's atom and accepted it by 1915. According to Reiche, it was Ladenburg the person who introduced him to Bohr's 1913 trilogy, and who influenced him the most in his interest in atomic physics. Reiche recalled having corresponded with Ladenburg about Bohr's atom around 1915 (Collection by Reiche, Fritz; AHQP, APS, M/f No. 1419-04-reiche-003: 7-8).

Ladenburg's support of Bohr's theory was not an exception. By the end of the war, Bohr's atomic model had won many followers, since it had extended its empirical basis and rested on better-established theoretical and mathematical grounds. ${ }^{54}$ In particular, Bohr's atomic model must have been a discussion topic in the scientific circles of Berlin during the last years of the war. Many physicists gathered in the city during the war, being part of different institutions devoted to military research, as for example Ladenburg's department in Berlin, and Nernst's and Fritz Haber's Institutes of the Kaiser-Wilhelm society. There was also Rubens' colloquium of experimental physics, which many physicists who had been demobilized in Berlin those years regularly attended. There the newest aspects of quantum physics were discussed, in particular Bohr's atomic model. Ruben's colloquium was led by Born in those years, who recalled that "it was the most brilliant collection of people I have ever directed. And there were arranged, of course, talks about the most modern discoveries" (AHQP, APS, M/f No. 1419-01-born-003: 4). Last but not least, the city hosted some important meetings of physicists, such as the series of lectures on quantum physics dedicated to Max Planck's 60 birthday, held on April 26th 1918 in the big auditorium of the Institute of Physics of the University of Berlin. It would be very surprising that Ladenburg had not taken part in some of these events while leading the department for sound ranging in Berlin.

\footnotetext{
${ }^{54}$ For a better explanation of how Bohr's atomic model gained further support, see (Kragh, 2012, 90-188). Though the earliest reactions had been critical or skeptical, from 1915 onwards the situation changed. To begin with, in 1916 Bohr's atom received support from James Franck's and Gustav Hertz's collision experiments between atoms and electrons (Franck, 1916a; Franck, 1916b). Yet it were Sommerfeld's 1916 contributions, already mentioned, which became the turning point for the general acceptance of Bohr's atomic model. There Sommerfeld generalized Bohr's model to elliptic orbits, proposed an alternative formulation of quantum conditions and used it to explain the so-called Stark effect and X-Ray spectroscopy (Sommerfeld, 1916a; Sommerfeld, 1916b; Kossel, 1916). On the basis of Sommerfeld's new theoretical and mathematical foundation, the empirical grounds of Bohr's model could be considerably enlarged, as in the case of the normal Zeeman effect, X-ray spectroscopy and the fine-structure splitting of hydrogen spectrum. Moreover, the Bohr-Sommerfeld quantum theory provided a unified explanation of simple collision phenomena, chemical insights and other properties of matter, such as radioactivity and magnetism. In all, and despite a few critical voices, the Bohr-Sommerfeld theory was generally accepted, used and discussed by 1918, above all in Germany, but also in the Netherlands and Sweden (Kragh, 2012, 90-188). Furthermore, by 1919 Bohr's atomic model was broadly transmitted in important textbooks devoted to atomic theory in Germany, most importantly, Sommerfeld's Atombau und Spektrallinien (Sommerfeld, 1919), which became to be known as the "Bible" of atomic theory for modern physicists (Eckert, 2013).
} 
Unsurprisingly, quantum theory became Ladenburg's focus of interest when he came back tor Breslau in 1919. At the beginning, Ladenburg's efforts were devoted to reorganize and make sense of existing experimental data in terms of the quantum theory. Already in March 1919, he gave a talk on quantum theory, entitled "Über die neuen Vorstellungen vom Atombau und der Molekülbildung", published one year later in Naturwissenschaften (Ladenburg, 1920a), in which Ladenburg built a consistent account of the chemical and physical properties of chemical elements, like the volume of atoms, their magnetization, color, weight, valence, and atomic number, together with the X-ray spectrum, on the grounds of Bohr's atomic model. The content of this papers reveals a great mastery of the new experimental and theoretical literature on atomic physics.

Soon afterwards Ladenburg published another contribution dealing with the quantum theory. The title was "Bericht über die Bestimmung von Plancks elementarem Wirkungsquantum h". The approach of the paper was rather original. Instead of using the famous proportionality relation $E=h \nu$ to understand new experimental features, Ladenburg did the opposite thing: he described the different ways in which experiments of already known "quantentheoretisch behandelten Erscheinungen" (Ladenburg, 1920b, 102) could be used to find empirical values of Planck's constant $h$, depending on the ways in which the quantum discontinuity manifested, namely, thermal radiation, monochromatic phenomena or threshold behaviors in light-matter interactions. His goal was to determine which was the most precise way to calculate the value of $h$ from experiments. Optical dispersion was obviously not mentioned. It was not deemed as a "quantentheoretisch behandelte Erscheinung" yet.

\subsection{Ladenburg's 1921 paper}

Optical dispersion was tackled in Ladenburg's third paper on quantum theory, published in 1921, with the title "Die quantentheoretische Deutung der Zahl der Dispersionselektronen" (Ladenburg, 1921). There Ladenburg put forward the first quantum interpretation of the classical parameter $N$, which referred to the number of dispersion electrons. As the title indicates, the topic of the paper was the physical meaning of $N$ in particular, and not optical dispersion in general.

\subsubsection{Ladenburg's approach}

Ladenburg's 1921 paper was at the same time a continuation of his two previous papers on quantum theory, a continuation of this work on optical dispersion prior to 1914, and a novel contribution to quantum theory. First, it was a continuation of his two 1920 papers because Ladenburg kept on analyzing and reorganizing existing phenomena in the light of quantum theory, in particular Bohr's atomic model. No novel data or newly discovered phenomena were discussed. Second, it was a continuation of Ladenburg's own research on dispersion before WWI because in 1921 he eventually answered the question he had raised before 1914: what is the physical meaning of the number $N$ of dispersion electrons? Furthermore, the strategy he followed and the conceptual machinery he used in 1914 to tackle the problem were the starting point of his 1921 paper. Third, it was a novel contribution to quantum theory because optical dispersion had never been considered as a quantum phenomenon before. According to Debye's and Sommerfeld's hybrid theories, optical dispersion continued to be a classical phenomenon, though taking place within quantum atomic and molecular structures, because dispersion continued stemming from the mechanism of resonance between light and matter. According to Ladenburg's 1921 reinterpretation of $N$, instead, optical dispersion should be regarded as a quantum phenomenon. To be 
sure, he did not put forward a new quantum theory of the phenomena that explained how light and matter interacted to give rise to optical dispersion. Yet, through Ladenburg's 1921 reinterpretation of $N$, optical dispersion came to be fully determined by quantum frequencies and transition probabilities, instead of resonance frequencies and the number of resonating electrons.

The gist of Ladenburg's argument was the identification of the energy $J_{E l}$ absorbed by $N$ classical resonating electrons with the energy $J_{Q}$ absorbed by $N^{\prime}$ Bohrian atoms performing quantum transitions, which implied the identification of transition frequencies with the frequencies at which dispersion electrons theoretically resonated. That is to say, Ladenburg connected two exclusive models of light-matter interactions. By equating the expression of $J_{E l}$ depending on $N$ to the expression of $J_{Q}$ depending on $N^{\prime}$, he obtained a new mathematical expression of $N$ depending on $N^{\prime}$ and the probabilities of quantum transitions.

The whole approach to the problem of dispersion electrons was very similar to Ladenburg's 1914 argumentation. In both cases, what was calculated was the amount of energy absorbed/emitted at spectral lines in terms of the number $N$ of dispersion electrons. In 1914, he equated this expression to an expression depending on purely empirical parameters, such as the luminance. In 1921, he equated two theoretical expressions: the energy absorbed/emitted by $N$ classical resonators and the energy absorbed/emitted by $N^{\prime}$ quantum atoms. In the first case, Ladenburg's goal was to calculate number of dispersion electrons from experiments. In the second case, it was to reinterpret $N$ in terms of Bohr's atomic model. Despite these differences, Ladenburg's energy argument and recourse to Planck's oscillators allowed him to avoid any discussion about the specific mechanism of light-matter interactions. In contrast, all theoreticians had so far approached dispersion by setting out the equations or motion of matter interacting with light.

Ladenburg's reasoning went in three steps. The first step was to find an expression of the energy absorbed by $N$ classical resonating electrons. This first step summarized the first two steps of his 1914 paper, as explained in Section 3.6.2. As in 1914, the trick was to identify the $N$ resonating electrons with $N$ Planck's resonators of the same frequency $\nu_{0}$, which allowed Ladenburg to identify the mean energy of one resonating electron with the mean energy $U$ of one Planck's resonator that was in equilibrium with the thermal radiation of the same frequency. ${ }^{55}$ This time Ladenburg was more straightforward and he did not even bother to justify the use of Planck's oscillators by arguing that emitted/absorbed radiation was thermal radiation. He simply assumed so.

Obviously, Ladenburg found the same expression as in 1914 for the energy $J_{E l}$ emitted by $N$ resonating electrons per unit time:

$$
J_{E l}=\frac{\pi e^{2}}{m} N u_{0}
$$

Due to thermal equilibrium, the amount of absorbed energy should be the same as the amount of emitted energy. As in 1914, Ladenburg emphasized the universal character of the above expression, since it was independent of the specific microscopic mechanism causing light absorption. ${ }^{56}$ To prove such a claim, he quoted his own 1914 paper and acknowledged Reiche's unpublished calculations on this matter between 1913 and 1915.

\footnotetext{
${ }^{55}$ The formula (11) relates the mean energy $U$ of one Planck's resonator to the spectral density $u_{u}$ of thermal radiation of the same frequency $\nu$. In 1921, Ladenburg added a factor 3 to this expression in order to take into account that resonators had three degrees of freedom, instead of only one. Therefore, Ladenburg wrote in 1921: $U=\frac{3 c^{3}}{8 \pi \nu^{2}} u_{\nu}$, instead of $U=\frac{c^{3}}{8 \pi \nu^{2}} u_{\nu}$, as in 1914. Nevertheless, this factor does not affect the final expression for $J_{E l}$ depending on $u_{\nu}$ and $N$.

${ }^{56}$ The above expression is a combination of formulas (12) and (13) of the paper.
} 
The second step was to calculate the amount of energy $J_{Q}$ absorbed by $N^{\prime}$ molecules performing quantum transitions. Obviously, this step was not in his 1914 paper. For this purpose he used the notion of transition probability, which Einstein had introduced in 1916 when trying to re-derive Planck's radiation law by other means (Einstein, 1916). Einstein had pictured a gas as molecules having two different quantum states between which electrons could jump. The gas was placed in a closed cavity with radiation, having a certain distribution $u_{\nu}$ of energy over frequencies. Einstein figured out three ways in which molecules could exchange energy with radiation: through spontaneous emission, absorption, or stimulated emission of light. The last kind of emissions was induced by the radiation field of the same frequency. Each frequency $\nu_{i k}$ was determined by Bohr's relation $E_{k}-E_{i}=h \nu_{i k}$, thus all processes of energy exchange between light and matter took place through quantum transitions between two sates, let's say $k$ and $i$. Quantum transitions took place from the state $k$ to the state $i$ for emission and from the state $i$ to the state $k$ for absorption. Einstein assumed a probability $d W$ that each kind of transition (spontaneous emission, stimulated emission, and absorption) occurred in an interval of time $d t$, depending on three postulated probability coefficients $a_{k i}, b_{k i}$, and $b_{i k}$, respectively. More specifically, Einstein established that: $d W=a_{k i} d t, d W=b_{k i} u_{\nu_{i k}} d t$ and $d W=b_{i k} u_{\nu_{i k}} d t$. The first coefficient was for spontaneous emission, the second for induced emission, and the third for absorption. To re-derive Planck's radiation Einstein assumed energy balance at thermal equilibrium, that is to say, the amount of radiation absorbed by molecules coincided with the amount of energy emitted by them, taking both spontaneous and stimulated emissions into account.

Following Einstein's argument, Ladenburg wrote that the total amount of energy emitted by $N_{k}$ molecules in the state $k$, per second, was:

$$
J_{Q}=h \nu_{i k} N_{k}\left(a_{k i}+b_{k i} u_{i k}\right)
$$

$\nu_{i k}$ being the frequency of transition and $u_{i k}$ the radiation density. In a situation of thermal equilibrium, this energy should coincide with the energy absorbed by $N_{i}$ molecules in the state $i$ :

$$
A_{Q}=h \nu_{i k} N_{i} b_{i k} u_{i k}
$$

$b_{i k}$ being the absorption probability coefficient. In such a situation of equilibrium, at temperature $T$, the number of molecules in the $k$ and $i$ states was related in the following way:

$$
\frac{N_{k}}{N_{i}}=\frac{g_{k}-\frac{E_{k}}{k T}}{g_{i}-\frac{E_{i}}{k T}},
$$

$g_{k}$ being the statistical weight of the state $k$ and $g_{i}$ of the state $i$. In order to fulfill the condition of thermal equilibrium, $b_{i k}$ and $a_{k i}$ should relate to each other in the following way, according to Einstein:

$$
b_{i k}=a_{k i} \frac{g_{k}}{g_{i}} \frac{c^{3}}{8 \pi \nu_{i k}^{2}} .
$$

Using these previous developments, Ladenburg wrote the following expression for the amount of energy absorbed through quantum transitions. Instead of expressing this quantity in terms of Einstein's absorption coefficient, he used the coefficient of spontaneous emission. This was a clever step that allowed him to relate dispersive 
phenomena to other optical phenomena involving emission, like fluorescence:

$$
J_{Q}=A_{Q}=N_{i} \frac{g_{k}}{g_{i}} a_{k i} \frac{c^{3}}{8 \pi \nu_{i k}^{2}} u_{i k} .
$$

There was no big novelty in this procedure. Ladenburg simply followed Einstein's reasoning very closely. For someone familiar with the literature on the quantum theory, Einstein's probability coefficients were a well-established concept that complemented Bohr's atomic picture. To begin with, Bohr had used the notion of transition probability for his Correspondence Principle in 1918 (Bohr, 1918). Other experimentalists had also had recourse of the same notion to interpret their data on the grounds of Bohr's atomic theory (Stern, 1919; Füchtbauer, 1920). In his second 1920 paper, Ladenburg presented Einstein's coefficients as the second axiom of the quantum theory, after Bohr's stationary states and quantum jumps (Ladenburg, 1920b, 101-102).

The third and last step of Ladenburg's argument was the riskiest and most original one. Ladenburg equated the amount of energy $J_{E l}$ absorbed by $N$ classical electrons (formula (21)) with the amount of energy $A_{Q}$ absorbed by $N_{i}$ molecules in the state $i$ (formula (26)). That is to say:

$$
\frac{\pi e^{2}}{m} N u_{0}=N_{i} \frac{g_{k}}{g_{i}} a_{k i} \frac{c^{3}}{8 \pi \nu_{i k}^{2}} u_{i k} .
$$

From the above-expression, one could obtain a new expression relating $N$ to $N_{i}$ :

$$
N=N_{i} \frac{g_{k}}{g_{i}} a_{k i} \frac{m c^{3}}{8 \pi^{2} e^{2} \nu_{i k}^{2}} .
$$

This expression allowed Ladenburg to relate parameters that had a meaning only in the classical framework (numbers of dispersion electrons $N$ ) to parameters that only made sense in a quantum framework (the absorption coefficient $a_{k i}$ and the ratio of statistical weights $\frac{g_{k}}{g_{i}}$ ). It was in this way that Ladenburg reinterpreted $N$ in terms of quantum theoretical notions.

This step had very deep implications, and implications of which Ladenburg was well aware. In order to obtain the formula (28), it was necessary to assume that the resonance frequency $\nu_{0}$ coincided with the transition frequency $\nu_{i k}$, so that $u_{0}=u_{i k}$. Yet both mechanisms, resonance and quantum jumps, were conceptually incompatible. This was indeed the whole point of the Debye-Sommerfeld dispersion theory. For their hybrid theory to work, Sommerfeld had to impose a strong restriction: dispersion (hence resonance) frequencies and spectral (hence transition) frequencies could not coincide. They should be very far away from each other. Otherwise the DebyeSommerfeld dispersion theory collapsed. It is not that Ladenburg was not aware of the conceptual problems between the mechanism of resonance and quantum jumps. Yet, at the same time he knew perfectly well that spectral and dispersion frequencies did experimentally coincide. Ladenburg's experiments on dispersion manifested precisely the situation Sommerfeld had theoretically excluded.

Ladenburg preferred not to delve into these conceptual problems. In order to do so, he regarded $N$ as an "experimental quantity, which did not have any physical meaning in the quantum theory" (Ladenburg, 1921, 454). This was a key point. To speak of $N$ as the number of dispersion electrons would have made no sense in the context of the quantum theory. Yet, if he considered $N$ as an empirical parameter, he could ascribe it a new physical meaning in terms of completely different physical quantities without facing the typical conceptual problems between resonance 
and quantum mechanisms. Ladenburg could not simply put on the same foot two conceptually incompatible pictures of light-matter interactions. Although Ladenburg was only concerned with the reinterpretation of $N$, the whole dispersion formula was implicitly taken for granted and considered an empirical formula, so that $\nu_{0}$ could be simply identified with $\nu_{i k}$. On a theoretical basis, $\nu_{0}=\nu_{i k}$ was unconceivable. Yet on an experimental basis, it was clear that the frequencies around which dispersion was anomalous, namely, $\nu_{0}$, coincided with the spectral frequencies, which were identified with transition frequencies $\nu_{i k}$.

The identification of dispersion frequencies with transition frequencies was thus a self-evident move for Ladenburg. Therefore it was just mentioned in a footnote, the third one of page 454 (Ladenburg, 1921, 454). In his interview with Thomas Kuhn in 1962, Reiche also mentioned that the coincidence of the frequencies in the denominator of the dispersion formula with the transition frequencies was something considered as self-evident by Ladenburg and himself in a paper they coauthored two years later (Collection by Reiche, Fritz; AHQP, APS, M/f No. 1419-04-reiche-003: 1011). As a matter of fact, in 1921 Reiche had just been appointed to Breslau and started to discuss again on quantum theory with Ladenburg. In 1923 both friends wrote a paper together, which was based on Ladenburg's 1921 contribution (Ladenburg and Reiche, 1923).

\subsubsection{The scope of Ladenburg's reinterpretation}

Ladenburg's reinterpretation did not only concern optical dispersion, but all optical phenomena sharing the same parameter $N$, namely, the so-called dispersive phenomena, such as the already mentioned magnetorotation of light polarization and other phenomena. ${ }^{57}$

The spread of spectral lines was another dispersive phenomenon that could be reinterpreted on the grounds of Ladenburg's 1921 paper. It was well known that spectral lines were never purely monochromatic, but they spread around a central frequency (see Fig. 10). From the point of view of classical electron theories, emission and absorption were explained on the basis of resonant electrons. Vibrating electrons absorbed light at their resonant frequencies because they were damped, that is to say, the amplitude of their vibrations decreased exponentially in time depending on a characteristic damping constant $\nu^{\prime}$. According to classical theories, ${ }^{58}$ the curve of light intensity around absorption lines was a function of $\nu$ and depended on the parameter $\nu^{\prime}$, and also the typical dispersion parameters, to wit the resonance frequencies $\nu_{i}$ and the strength of dispersion $K_{i}$ (which was proportional to the number of dispersion electrons according to formula (3)). ${ }^{59}$ As a consequence, the intensity curve was also proportional to $N$, as the dispersion formula, and experiments of the spread of absorption lines could also be used to count the number of dispersion electrons at different spectral lines. This is what the German experimentalist Christian Füchtbauer and his collaborators did before WWI in their experimental program called "quantitative spectroscopy" (Füchtbauer and Hofmann, 1913): to count dispersion electrons from experiments of the spread of spectral lines. ${ }^{60}$

In the light of Bohr's atomic model, the results of the "quantitative spectroscopy" had also to be reinterpreted. Füchtbauer himself did so in 1920 (Füchtbauer, 1920). According to him, the energy absorbed during absorption, which was proportional to the area under the intensity curve, should not be proportional to the number of

\footnotetext{
${ }^{57}$ See Section 3.2 of this paper.

${ }^{58}$ See Voigt's textbook (Voigt, 1908).

${ }^{59}$ For simplicity sake, dispersion formula was used by approximating $\nu^{\prime}$ to zero.

${ }^{60}$ For more information about Füchtbauer's "quantitative spectroscopy," see (Jordi Taltavull, 2019, 342-363).
} 


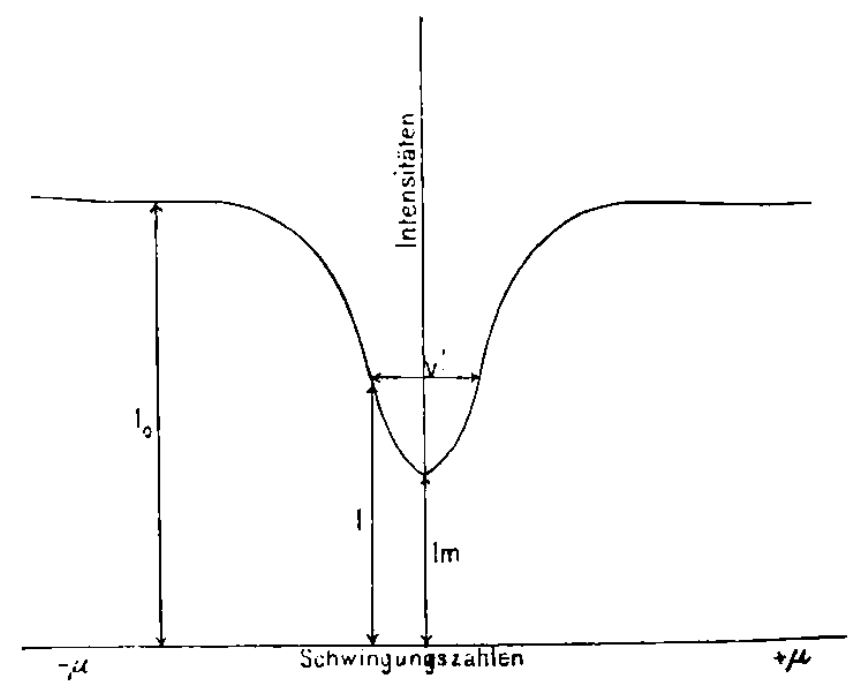

Fig. 10. Theoretical intensity curve at one absorption line, with significative parameters. $J_{0}$ is the intensity without absorption. $J_{m}$ is the intensity at the position of maximum absorption, namely, minimum intensity, whose position corresponds to the theoretical spectral frequency. The intensity $J$ would be related to $J_{m}$ according to the following expression, $\frac{J}{J_{0}}=e^{-q}$, given that $\frac{J_{m}}{J_{0}}=e^{-2 q}$. The damping constant $\nu^{\prime}$ would be the width of the spectral curve, when the intensity was $J$ (Füchtbauer and Hofmann, 1913, 100).

dispersion electrons $N$ resonating at the absorption frequency, but to the number of quantum transitions of the same frequency $\nu_{i k}$. The coincidence of the quantum frequency $\nu_{i k}$ with the old resonance frequency was self-evident. In turn, the number of quantum absorption transitions was proportional to Einstein's probability coefficient $b_{i k}$ and to the number $N_{i}$ of molecules in the lower state $i$. By comparing the area under the intensity curve with the theoretical value of the energy absorbed by $N_{i}$ quantum molecules transiting from the state $i$ to the state $k$, Füchtbauer found an expression that related the measurable parameter $\nu^{\prime}$ to the quantum parameters $b_{i k}$ and $N_{i}$. He did not explain how monochromatic transitions could theoretically give rise to the spread of frequencies, yet he could reinterpret his experimental results in the light of the quantum theory.

As a matter of fact, Ladenburg knew of Füchtbauer's 1920 quantum reinterpretation of the spread of spectra lines, and he even quoted it at the very beginning of his 1921 paper. Nevertheless, Ladenburg's results were much more general than Füchtbauer's. Füchtbauer's conclusions were limited to the spread of absorption lines. Ladenburg's conclusions, instead, applied to all classical phenomena depending on $N$ resonating electrons, including optical dispersion, magnetooptical phenomena, and the spread of spectra lines.

Ladenburg's 1921 reinterpretation could also be applied to phenomena involving emission at spectral lines. This is because Ladenburg had re-expressed $N$ in terms of the coefficient of spontaneous emission $a_{k i}$, instead of the coefficients of absorption and induced emission. It was the case of the emission of fluorescence light at transition frequencies. It was commonplace that the intensity of light emitted during fluorescence gradually decreased over a small period of time. Such a decay time could not be explained on the basis of instantaneous transitions. In 1919, Max Volmer and Otto Stern suggested that the decay time $\tau$ of fluorescence should be interpreted as the mean life of $N_{k}$ molecules in the state $k$. They did so by following Einstein's comparison of the spontaneous decay of $N_{k}$ molecules from the state $k$ to the state $i$ 
to a radioactive process (Einstein, 1916, 386):

$$
N_{k}^{\prime}=N_{k} e^{-\frac{t}{\tau}}
$$

in which $N_{k}$ was the initial number of molecules in the state $k$ and $N_{k}^{\prime}$ was the number of molecules still in the state $k$ at time $t$. Ladenburg was aware of Stern and Volmer's contribution and identified $\tau$ with the reciprocal of $a_{k i}$, namely, $\frac{1}{a_{k i}}=\tau$. This was the case in which only one transition was possible, between the state $k$ and the state $i$. Since Ladenburg recognized that atoms in one quantum state could transit to many different states, $i, h, g, \ldots$ he related the mean life of the atom in state $k$ to the reciprocal of the sum $a_{k}=a_{k i}+a_{k h}+a_{k g}+\cdots$.

Altogether, Ladenburg's 1921 reinterpretation of the number of dispersion electrons did not only concern optical dispersion, but all optical phenomena of emission and absorption that were caused by resonant electrons according to classical theories.

\subsubsection{Ladenburg's reinterpretation at work}

Despite the far-reaching scope of Ladenburg's reinterpretation of $N$, no quantum theory of dispersive phenomena was put forward. How instantaneous and monochromatic transitions could give rise to the spread of spectral lines around the quantum frequency, the continuous dispersion of light, and the gradual rotation of light polarization, remained a riddle. Ladenburg put forward a possible explanation of anomalous dispersion in the new scenario, but he made clear that no quantum theory of anomalous dispersion existed yet. In particular, he suggested that the radiation field interacted with molecules by modifying their stationary states, so that the emission and absorption processes did not always occur exactly at $\nu_{i k}$, but also at neighboring frequencies. In addition to absorption, such a modification of stationary states should also be associated to a change of velocity of light.

Rather than a new theory of dispersive phenomena, what interested Ladenburg the most was to use his reinterpretation of $N$ on the basis of already existing experimental data in order to obtain empirical values of the probability coefficients of specific materials, in particular monoatomic gas of hydrogen and alkali metals. Ladenburg combined experimental data with already known information on quantum states and possible transitions, as in Figure 11 for the monoatomic gas of hydrogen. For example, as already told in Section 3.2, in 1911 Ladenburg obtained the following ratio for the number of dispersion electrons vibrating at two frequencies of the Balmer series, $\frac{N_{H_{\alpha}}}{N_{H_{\beta}}} \approx 4$. In 1911, he interpreted this data as if the number of dispersion electrons resonating at the spectral frequency $H_{\alpha}$ was four times the number of electrons resonating at the spectral frequency $H_{\beta}$. In 1921, he used the same data to claim that $\frac{a_{\alpha}}{a_{\beta}} \approx 4$, namely, that the probability coefficient for a transition of the spectral frequency $H_{\alpha}$ was four times the probability coefficient for a transition of the spectral frequency $H_{\beta}$. For such a claim, it was necessary to assume that the states from which electrons jumped to a higher state were the same both for $H_{\alpha}$ and $H_{\beta}$. Ladenburg could assume so because it was already known that the spectral line $H_{\alpha}$ was due to electron jumps between orbits with quantum number 2 and quantum number 3 , whereas the spectral line $H_{\beta}$ was due to electron jumps between orbits with quantum number 2 and quantum number 4 .

Further information about monoatomic gas of hydrogen could be obtained if another kind of experiments were also discussed: canal rays (Stark, 1916; Wien, 1919), namely, the emission of light by this gas when it was excited by accelerated ions. The decay time of the intensity of canal rays at different spectral frequencies provided information about the mean life of the corresponding states from which quantum 


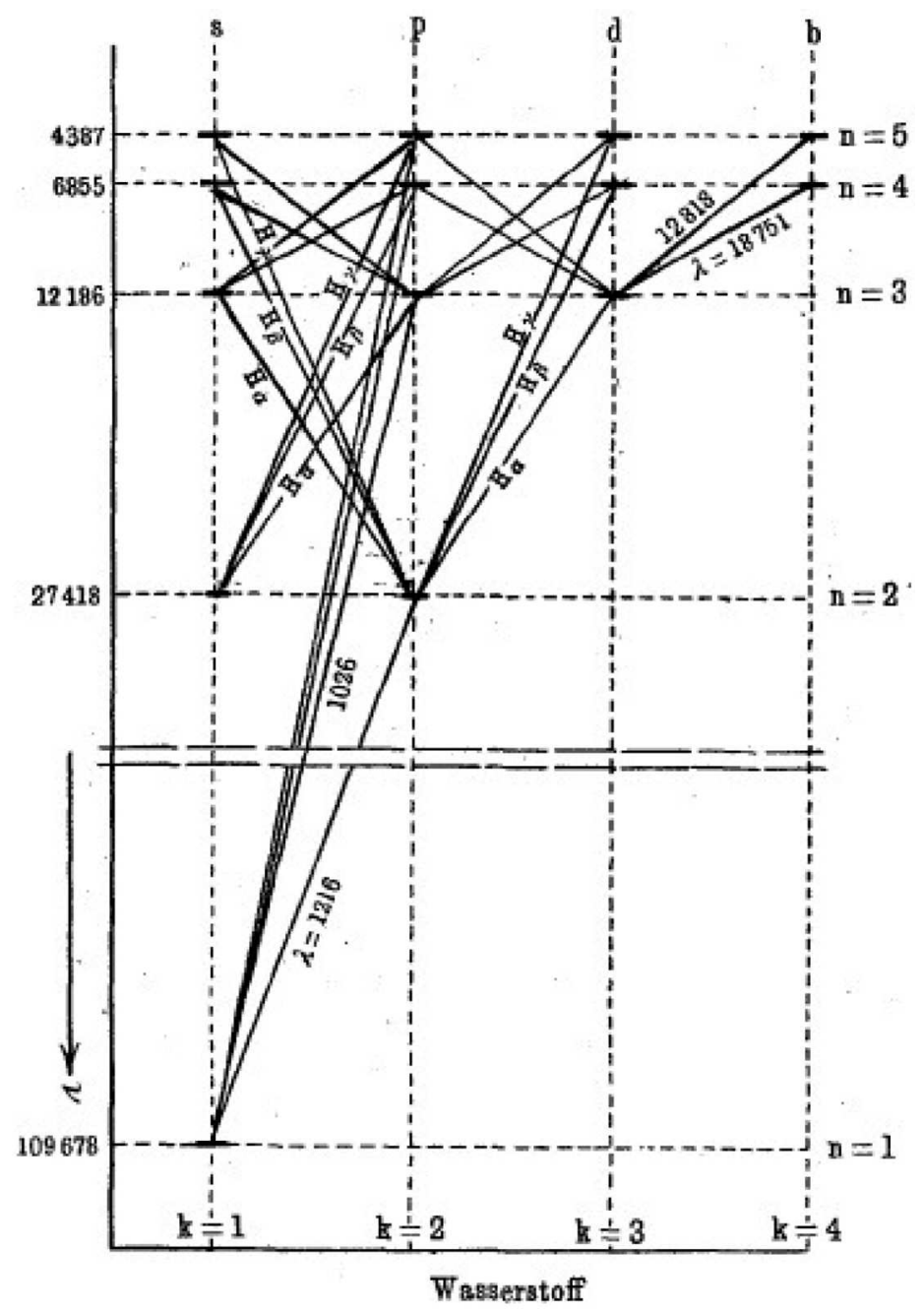

Fig. 11. Hypothetical map of allowed quantum transitions in connection to the stationary states of hydrogen (Ladenburg, 1921, 458).

transitions took place, and therefore to the corresponding probability coefficients for spontaneous emission. A more complete picture about transition probabilities could thereby be provided. Eventually, the same kind of arguments was applied to other materials, in particular alkali metals, on the basis of Wood's, Bevan's, Loria's, and Füchtbauer's experimental data.

Hence the old problem of dispersion electrons, namely, the decreasing of number of dispersion pro increasing frequency of the spectral line, had been simply reformulated: it was a decreasing of the transition probability for increasing frequency. A new explanation of this feature was possible on the grounds of Bohr's theory: the transition probability increased because the number of possible transitions from the initial state increased, as in the example with the monoatomic gas of hydrogen. For experiments involving the intensity decay of emitted light, a reformulation of the problem was also required: the intensity decay did not reflect the decay time of classical resonators $\tau$, but the mean life of quantum atoms and molecules in one specific state $k$, in 
particular, $\frac{1}{\tau}=a_{k i}+a_{k h}+a_{k g} \cdots$. Furthermore, if practically all molecules found themselves in one state $k$ and only one transition from that state was possible, an analogy could be stated: an atom in the initial state $k$ resembled a classical resonator whose decay time $\tau$ coincided with the mean life of that atom in state $k$, hence $\tau=\frac{1}{a_{k i}}$ and whose frequency coincided with the transition frequency. ${ }^{61}$

All in all, Ladenburg had found a unified way to speak of all these phenomena in terms of quantum physics by changing the physical meaning of old parameters, without changing the original mathematical formulations and without having developed any new quantum theory of the phenomena. Remarkably, Ladenburg continued using the old parameter $N$ to present experimental results. Only after having calculated $N$ he used the formula (28) to find a quantum reinterpretation of it. Thus both pictures, the classical and quantum one, were in play. The first one was to describe experimental results, the second one was to provide parameters with "quantum meaning" (Ladenburg, 1921, 464), to reinterpret experiments in the "quantum language" (Ladenburg, 1921, 466). The same duality appeared in a paper published in the same year by Ladenburg and his collaborator Rudolph Minkowski. The two men first calculated the values of $N$ from experiments, which they presented "in der Sprache der Elektrontheorie" (Ladenburg and Minkowski, 1921, 163), and only afterwards they reinterpreted them "quantentheoretisch" (Ladenburg and Minkowski, 1921, 163). ${ }^{62}$

\subsection{The origin of Ladenburg's 1921 idea}

Now the question remains how Ladenburg could have figured out such a reinterpretation of optical parameters.

Füchtbauer's 1920 quantum reinterpretation of the spread of spectral lines and Stern and Volmer's 1919 quantum reinterpretation of the intensity decay of fluorescence could have been sources of inspiration for Ladenburg. Both were acknowledged by Ladenburg in his 1921 paper. Both Ladenburg's and Füchtbauer's arguments were based on the proportionality between energy absorbed/emitted and number of dispersion electrons. Moreover, Ladenburg re-expressed $N$ in terms of the emission coefficient $a_{k i}$, instead of the absorption coefficient $b_{i k}$, because in this way he could connect dispersive phenomena to the intensity decay of fluorescence. Yet Ladenburg's approach was much more general, by encompassing not only one phenomenon, but all phenomena caused by $N$ resonating electrons in the classical theory.

Ladenburg also acknowledged the advice of his Breslau colleagues Reiche and Kohn. As is well known, Reiche mastered quantum theory and had just written a textbook entitled "Die Quantentheorie, ihr Ursprung und ihre Entwicklung" (Reiche, 1921). Nevertheless, dispersion theories were tackled in the old way in Reiche's book. Reiche reported on Debye's and Sommerfeld's theory of dispersion. He criticized the fact that their model of molecular hydrogen presented several pitfalls, but he did not expand on the fact that resonance frequencies did not coincide with spectral frequencies. Actually, Reiche always emphasized that the quantum reinterpretation of the number $N$ of dispersion electrons in 1921 was Ladenburg's own idea. During his interview with Kuhn in 1962, Reiche referred to it as "the idea of Ladenburg". In speaking about Ladenburg and Reiche's 1923 joint paper, Reiche told Kuhn that the

\footnotetext{
${ }^{61}$ This was the case of alkali metals. The first spectral line corresponds to emissions from the state $2 p$. Only one transition from this state is allowed.

${ }^{62}$ In this paper, the two men published new data of the rotation of light polarization of sodium gas in the neighborhood of the two D-lines. The goal was to measure the absolute values of the parameter $N$ at the two spectral D-lines and to compare them with the total number of sodium molecules, in order to know the proportion of emitting molecules in the gas. A new experimental method was used in order to control the temperature of the sample and therefore to know the absolute value of atoms present in the sample.
} 
1923 paper "uses already obviously this previous paper by Ladenburg" (Collection by Reiche, Fritz; AHQP, APS, M/f No. 1410-04-reiche-004: 13). ${ }^{63}$

Furthermore, Ladenburg was more than simply a good experimentalist. As Reiche explained Kuhn in the same interview about Ladenburg: "He was, as far as I understand, a very good experimental man, but he was one of the men who could make, let me say, easy theoretical work" (Collection by Reiche, Fritz; AHQP, APS, M/f No. 1410-04-reiche-004: 10). In the same line, his colleague Kohn defined Ladenburg as the man with "quantum mechanical intuition" in the Breslau group. In the years after 1921, Reiche, Kohn, Ladenburg, and Eucken, used to discuss physical issues. In her interview with Kuhn, Kohn talked about the group in the following way:

She also indicates that he [Eucken], she, Ladenburg and Reiche formed a group at Breslau which always discussed their problems together. It was through this group that most of her own contact with the development of quantum physics came. In the group she again speaks of Ladenburg as the leader in quantum mechanical intuition (Collection by Kohn, Hedwig; AHQP, APS, M/f No. 1419-03-kohn-002: 01).

Ladenburg also corresponded with Bohr in those years. Between 1920 and 1924, Bohr and Ladenburg exchanged more than a dozen letters, in which Ladenburg showed his big interest in and a high mastery of Bohr's latest theoretical developments. The first of these letters was sent by Ladenburg on 7th May 1920. There, Ladenburg expressed his gratitude for Bohr's exciting lecture and ensuing discussion at the Bigwig Kolloquia in Berlin-Dahlem, held on the 28th April. Ladenburg did not miss this occasion to ask Bohr for a copy of his 1918 papers, in which Bohr presented his correspondence principle (Collection by Bohr, Niels; AHQP, BSC, M/f No. 4). Most probably Ladenburg had met Bohr at the Bigwig Kolloquia for the first time (James, 2011, 45), where Bohr gave a series of lectures on "Die Serienspektra der Elemente".

On June 25, 1921, Ladenburg corresponded with Bohr about optical dispersion for the first time. In this letter, Ladenburg highlighted the great similarity between certain reflections of Bohr on optical dispersion, published in the early 1921, and Ladenburg's own 1921 contribution. Bohr's reflections were the ones appearing in his famous 1916 withdrawn paper mentioned in Section 4.2, which was first published in 1921 in a German translation of Bohr's works on atomic theory (Bohr, 1921). There, Bohr mentioned the experiments of dispersion in the neighborhood of spectral lines as the strongest objections to Debye's and Sommerfeld's dispersion theories, he claimed that dispersion should somehow also depend on quantum transitions, and he suggested a possible proportionality between the numerator of the dispersion formula and the tendency of electrons to jump from one orbit to the other. ${ }^{64}$ This last point called Ladenburg's attention, for it resembled Ladenburg's suggested proportionality between $N$ and Einstein's coefficients. Bohr might have sent Ladenburg a copy of this recollection of his works in the early 1921:

Ganz besonders hat mich Ihre Bemerkung über den Zusammenhang zwischen Dispersion u. Übergangswahrscheinlichkeit (Abh. X. s.141) [This quotation refers to the German translation of Bohr's 1916 withdrawn paper, published in 1921] interessiert, da ich mich mit dieser Frage viel beschäftigt habe und zu Resultaten gekommen bin, die Ihrer Vermutung in gewisser Beziehung entsprechen (Z. f. Phy. 4, 451) (Collection by Bohr, Niels; AHQP, BSC, M/f No. 4).

\footnotetext{
${ }^{63}$ Quoted more extensively in (Duncan, 2007a, 582-583).

${ }^{64}$ At that moment, Bohr could not speak about probabilities of transitions yet. Einstein's paper appeared in 1916-1917.
} 
Certainly Bohr's remark could have influenced Ladenburg's ideas, yet it seems that Ladenburg perceived this similarity only a posteriori, after having sent off his paper for publication, already on February 5th 1921.

All in all, the fact that Ladenburg, and not a pure theoretician, came up with the idea of theoretically reinterpreting $N$ depends on several aspects: Ladenburg's specific object of study (anomalous dispersion and spectral lines), the specific related questions (the number of dispersion electrons), his own strategy to solve them (energy argument), the conceptual resources he availed himself of (Planck's resonators), some inspiration sources (Füchtbauer's and Stern and Volmer's, apart from Bohr's and Einstein's contributions), and his combined experimental and theoretical skills.

More specifically, Ladenburg's specific object of analysis (anomalous dispersion around the spectral lines) was simply excluded from Debye's and Sommerfeld's dispersion theory, which was only valid for normal dispersion. The questions that Ladenburg, on the one hand, and Debye and Sommerfeld, on the other hand, aimed at answering, were also very different. Debye's and Sommerfeld's question was how to theoretically include the mechanism of resonance within Bohr's quantum model of atom, without conceptual inconsistencies. Their strategy was to write down the possible equations of motion for electrons. They only used the empirical data that was convenient to them. Therefore anomalous dispersion was simply excluded from their conceptual approach. Experiments of anomalous dispersion around spectral lines were, instead, Ladenburg's starting point. In this context, he could not elude the evidence that resonance and dispersion frequencies were the same. The interpretive problem Ladenburg was tackling, namely, the decrease of dispersion electrons for increasing spectral frequencies, could not even be framed in the context of Debye's and Sommerfeld's theories. Ladenburg's strategy ever since 1914 was precisely to avoid motion equations, and to calculate the absorbed and emitted energy by resorting to abstract tools such as Planck's resonators. In 1921, Ladenburg simply combined the arsenal of conceptual resources he deployed before 1914 with Bohr's atomic model. Since issues of conceptual consistency were shifted to the background, differently from Debye's and Sommerfeld's concerns, Ladenburg found in Bohr's atomic model a new interpretive solution, instead of a problem. Obviously, Füchtbauer's and Stern and Volmer's reinterpretations of the spread of spectral lines and fluorescence, respectively, could have inspired Ladenburg, but it was on the grounds of his previous work on dispersion that he could embrace these contributions and accommodate them into his own argumentative strategy. In this case, Ladenburg's "quantum theoretical intuition" was not uniquely due to his theoretical skills, but to his ability to frame particular experimental problems in appropriate conceptual terms and to combine experimental practice with easy theoretical developments to solve them.

Acknowledgements. I am in debt with Prof. Dr. Tilman Sauer at the Johannes-Gutenberg University of Mainz for his exhaustive and constructive reviews of the paper, productive discussions and steady support in the last years. I would also like to thank Prof. Dr. Jürgen Renn at the Max Planck Institute for the History of Science of Berlin for his support, inspiring discussions and suggestions that helped shape my dissertation, on which this paper is based. I am also grateful to Michel Janssen at the University of Minnesota for having shared with me his knowledge on the topic and having motivated me to write this paper. Finally, I would also like to thank all members of the History of Quantum Physics Group at the Max Planck Institute for the History of Science of Berlin for invaluable intellectual exchange during the time I was there writing my dissertation. 
Open Access This is an open access article distributed under the terms of the Creative Commons Attribution License (https://creativecommons.org/licenses/by/4.0), which permits unrestricted use, distribution, and reproduction in any medium, provided the original work is properly cited.

Publisher's Note The EPJ Publishers remain neutral with regard to jurisdictional claims in published maps and institutional affiliations.

\section{References}

Aaserud, Finn and John Heilbron. 2013. Love, Literature and the Quantum Atom. Niels Bohr's Trilogy Revisited. Oxford University Press, Oxford.

Arabatzis, Theodore. 2006. Representing electrons. A biographical approach to theoretical entities. University of Chicago Press, Chicago.

Badino, Massimiliano. 2009. The odd couple: Boltzmann, Planck and the application of statistics to physics (1900-1913). Ann. Phys. 9: 81-101.

Badino, Massimiliano. 2015. The bumpy road. Max Planck from radiation theory to the quantum (1896-1906). Springer, New York.

Bergmann, Ludwig. 1958. Clemens Schaefer zum 80. Geburtstag. Die Naturwissenschaften 45: 121.

Bevan, P. V. 1910. Dispersion of light by potassium vapour. Proc. R. Soc. Lond. A 84: 209-225.

Blu, Alex, Martin Jähnert, Christoph Lehner, and Jürgen Renn. 2017. Translation as heuristics: Heisenberg's turn to matrix mechanics. Stud. Hist. Philos. M. P. 60: 3-22.

Bohr, Niels. 1913a. On the constitution of atoms and molecules (Part I). Philos. Mag. 26: $1-25$.

Bohr, Niels. 1913b. On the constitution of atoms and molecules (Part II). Philos. Mag. 26: 476-502.

Bohr, Niels. 1913c. On the constitution of atoms and molecules (Part III). Philos. Mag. 26: 857-875.

Bohr, Niels. 1918. On the quantum theory of line-spectra. Part I: on the general theory. Det Kongelige Danske Videnskabernes Skrifter, Naturvidenskabelig og Mathematisk Afdeling 8: $1-36$.

Bohr, Niels. 1921. Abhandlungen über Atombau aus den Jahren 1913-1916. Autorisierte deutsche Übersetzung mit einem Geleitwort von N. Bohr (übersetz von Dr. Hugo Stintzing). Vieweg und Sohn, Braunschweig.

Born, Max. 1975. Mein Leben. Die Erinnerungen des Nobelpreisträgers. Nymphenburger, Munich.

Born, Max. 1978. My life: recollections of a Nobel laureate. Charles Scribner's Sons, New York.

Buchwald, Jed Z. 1985. From Maxwell to microphysics. Aspects of electromagnetic theory in the last quarter of the nineteenth century. University of Chicago Press, Chicago.

Campbell, William W. 1895. Stars whose spectra contain both bright and dark hydrogen lines. Astrophys. J. 2: 177-183.

Christiansen, Christian. 1870. Über die Brechungsverhältnisse einer weingeistigen Lösung der Fuchsins; brieflicher Mittheilung. Ann. Phys. 217: 479-480.

Christiansen, Christian. 1871. Über das Brechungsverhältnis des Fuchsins. Ann. Phys. 219: $250-259$.

Darrigol, Olivier. 1992. From c-numbers to q-numbers: the classical analogy in the history of quantum theory. University of California Press, Berkeley.

Darrigol, Olivier. 2000. Electrodynamics from Ampre to Einstein. Oxford University Press, Oxford.

Darrigol, Olivier. 2001. The historian's disagreements over the meaning of Planck's quantum. Centaurus 43: 219-239. 
Darrigol, Olivier. 2012. A history of optics: from Greek antiquity to the nineteenth century. Oxford University Press, Oxford.

Debye, Peter. 1915. Die Konstitution des Wasserstoff-Moleküls. Sitzungsberichte der Mathematisch-Physikalischen Klasse der Königlich Bayerischen Akademie der Wissenschaften zu München: 1-26.

Dresden, Max. 1987. H. A. Kramers: Between Tradition and Revolution. Springer, New York.

Drude, Paul. 1893. Über die Beziehung der Dielectricitätsconstanten zum optischen Brechungsexponenten. Ann. Phys. 284: 536-545.

Drude, Paul. 1894. Physik des Athers auf elektro-magnetischer Grundlage. F. Enke, Stuttgart.

Drude, Paul. 1899. Zur Theorie der magneto-optischen Erscheinungen. Verh. Dtsch. Phys. Ges. 1: 107-116.

Drude, Paul. 1900. Lehrbuch der Optik. Hirzel., Leipzig.

Drude, Paul. 1904a. Optische Eigenschaften und Elektrontheorie. I. Teil Ann. Phys. 319: 677-725.

Drude, Paul. 1904b. Optische Eigenschaften und Elektrontheorie. II. Teil Ann. Phys 319: 936-961.

Drude, Paul. 1906. Lehrbuch der Optik, 2nd edn. Hirzel, Leipzig.

Duncan, Anthony and Michel Janssen. 2007. On the verge of Umdeutung in Minnesota: Van Vleck and the correspondence principle. Part one. Arch. Hist. Exact Sci. 61: 553-624.

Duncan, Anthony and Michel Janssen. 2007. On the verge of Umdeutung in Minnesota: Van Vleck and the correspondence principle. Part two. Arch. Hist. Exact Sci. 61: 625-671.

Ebert, Hermann. 1903. Die anomale Dispersion der Metalldämpfe. Phys. Z. 4: 473-476.

Eckert, Michael. 2013. Arnold Sommerfeld: Science, Life and Turbulent Times 1868-1951. Springer, Berlin.

Eckert, Michael and Karl Märker (eds.). 2000. Arnold Sommerfeld: Wissenschaftlicher Briefwechsel. Band I: 1892-1918. GNT-Verlag and Deutsches Museum, Munich.

Einstein, Albert. 1916. Strahlungs-emission und -absorption nach der Quantentheorie. Verh. Dtsch. Phys. Ges. 18: 318-323.

Einstein, Albert. 1917. Zur Quantentheorie der Strahlung. Phys. Z. 18: 121-128.

Epstein, Paul. 1916. Über die spezifische Wärme des Wasserstoffs. Verh. Dtsch. Phys. Ges. 18: $398-413$.

Epstein, Paul. 1922. Die Störungsrechnung im Dienste der Quantentheorie. I. Kritische Bemerkungen zur Dispersionstheorie. Z. Phys. 9: 92-110.

Franck, James and Gustav Hertz. 1916. Über Kinetik von Elektronen und Ionen in Gasen. Phys. Z. 17: 409-416.

Franck, James and Gustav Hertz. 1916. Über Kinetik von Elektronen und Ionen in Gasen. Phys. Z. 17: 430-440.

Füchtbauer, Christian. 1920. Die Absorption in Spektrallinien im Lichte der Quantentheorie. Phys. Z. 21: 322-324.

Füchtbauer, Christian and W. Hofmann. 1913. Über Maximalintensität, Dämpfung und wahre Intensitätsverteilung von Serienlinien in Absorption. Ann. Phys. 348: 96-134.

Gearhart, Clayton A. 2002. Planck, the quantum, and the historians. Phys. Perspect. 4: $170-215$.

Geiger, Ludwig. 1907. Über die Begleiterscheinung des inversen longitudinalen Zeemaneffektes. Ann. Phys. 328: 758-794.

Gouy, Louis G. 1879. Recherches photométriques sur les flammes colorées. Ann. Chim. Phys. 18: $5-101$.

Heilbron, John L. 1967. The Kossel-Sommerfeld Theory and the Ring Atom. Isis 58: 450585 .

Heilbron, John L. and Thomas Kuhn. 1969. The Genesis of the Bohr Atom. Historical Studies in the Physical Sciences 1: 211-290.

Helmholtz, Hermann von. 1875. Zur Theorie der anomalen Dispersion. Ann. Phys. 230: $582-596$. 
Helmholtz, Hermann von. 1892. Electromagnetische Theorie der Farbenzerstreuung. Sitzungsberichte der Königlich Preussischen Akademie der Wissenschaften zu Berlin: 1093-1109.

Helmholtz, Hermann von. 1893. Electromagnetische Theorie der Farbenzerstreuung. Ann. Phys. 284: 389-405.

Hentschel, Klaus. 1991. Julius und die anomale Dispersion: Facetten der Geschichte eines gescheiterten Forschungsprogrammes. Studien aus der Philosophischen Seminar 3, Universität Hamburg 6: 1-192.

Hentschel, Klaus and Ann M. Hentschel (eds.). 1996. Physics and national socialism. An anthology of primary sources. Birkhäuser, Basel.

Hilbert, David. 1904a. Grundzüge einer allgemeinen Theorie der linearen Integralgleichungen [erste Mitteilung]. Nachrichten von der Gesellschaft der Wissenschaften zu Göttingen, Mathematisch-Physikalische Klasse 1904: 49-91.

Hilbert, David. 1904b. Grundzüge einer allgemeinen Theorie der linearen Integralgleichungen [zweite Mitteilung]. Nachrichten von der Gesellschaft der Wissenschaften zu Göttingen, Mathematisch-Physikalische Klasse 1904: 213-259.

Hoyer, Ulrich. 1981. Work on atomic physics (1912-1917). In Niels Bohr Collected Works, Volume 2, ed. León Rosenfeld. North-Holland Publishing Company, Amsterdam.

James, Jeremiah, Thomas Steinhauser, Dieter Hoffmann, and Bretislav Friedrich. 2011. One hunderd years at the intersection of chemistry and physics. The Fritz Haber Institute of the Max Planck Society 1911-2011. Walter de Gruyter GmbH, Berlin.

Jammer, Max. 1966. The conceptual development of quantum mechanics. McGraw-Hill, New York.

Janssen, Michel. 2019. Arches and Scaffolds: bridging continuity and discontinuity in theory change. In Beyond the mem. Articulating dynamic structures in cultural evolution eds. Alan Love and William Wimsatt, 95-199. University of Minnesota Press, Minneapolis.

Frank B. Jewett. 1902. A new method of determining vapour-density of metallic vapours, and an experimental application to the cases of sodium and mercury. Philos. Mag. Series 6 4: 546-554.

Jordi Taltavull, Marta. 2013a. Challenging the boundaries between classical and quantum physics: the case of optical dispersion. In Traditions and transformations in the history of quantum physics, eds. Katzir, Shaul, Christoph Lehner, and Jürgen Renn, 29-59. Max Planck Research Library for the History and Development of Knowledge, Berlin.

Jordi Taltavull, Marta. 2013b. Sorting things out: Drude and the foundations of classical optics. In Research and pedagogy: a history of quantum physics through its textbooks, eds. Badino, Massimiliano and Jaume Navarro, 27-68. Max Planck Research Library for the History and Development of Knowledge, Berlin.

Jordi Taltavull, Marta. 2016. Transmitting knowledge across divides: optical dispersion from classical to quantum physics. Hist. Stud. Nat. Sci. 46: 313-359.

Jordi Taltavull, Marta. 2019. Transformation of optical knowledge from 1870 to 1925: optical dispersion between classical and quantum physics. Thesis. Humboldt Universität zu Berlin, Berlin.

Julius, Willem H. 1900. Solar Phenomena, considered in connection with anomalous dispersion of light Astrophys. J. 12: 185-200.

Jungnickel, Christa and Russel K. McCormmach. 1986. Intellectual mastery of nature: theoretical physics from Ohm to Einstein. II. The now mighty theoretical physics, 1870-1925. Chicago University Press, Chicago.

Ketteler, Eduard. 1876. Versuch einer Theorie der (anomalen) Dispersion des Lichtes in einfach und doppelt brechenden Mitteln Repertorium der Experimentalphysik, der physikalischen Technik und der astronomischen Instrumentenkunde 12: 322-361.

Ketteler, Eduard. 1879. Das Dispersionsgesetz. Ann. Phys. 243: 658-670.

Ketteler, Eduard. 1883. Optische Controversen. Ann. Phys. 254: 287-421.

Ketteler, Eduard. 1885. Theoretische Optik gegründet auf das Bessel-Sellmeier'sche Princip. Vieweg und Sohn, Braunschweig.

Koch, John. 1913. Über die Dispersion des Lichtes in gasförmigen Körpern innerhalb des ultravioletten Spektrums. Archiv für Matematik, Astronomi och Fysik 9: 1-11. 
Kohn, Hedwig. 1914. Über das Wesen der Emission der in Flammen leuchtenden Mettaldämpfe. Ann. Phys. 349: 749-782.

Konno, Hiroyuki. 1993. Kramers' negative dispersion, the virtual oscillator model, and the correspondence principle. Centaurus 36: 117-166.

Kopfermann, Hans. 1952. Rudolf Ladenburg. Die Naturwissenschaften 39: 25-26.

Kossel, Walther. 1916. Über Molekülbildung als Folge des Atombause. Ann. Phys. 354: 229-362.

Kragh, Helge. 1985. The fine structure of hydrogen and the gross structure of the physics community, 1916-1926. Historical Studies in the Physical Sciences 16: 67-125.

Kragh, Helge. 2012. Niels Bohr and the quantum atom. The Bohr model of atomic structure 1913-1925. Oxford University Press, Oxford.

Kuhn, Thomas S. 1978. Black-body theory and the quantum discontinuity, 1894-1912. Oxford University Press, Oxford.

Kundt, August. 1871a. Über die anomale Dispersion der Körper mit Oberflächenfarben. Ann. Phys. 218: 163-171.

Kundt, August. 1871b. Über anomale Dispersion. Ann. Phys. 219: 259-269.

Kundt, August. 1871c. Über anomale Dispersion. Ann. Phys. 220: 128-137.

Kundt, August. 1872. Über anomale Dispersion. Ann. Phys. 221: 67-80.

Kundt, August. 1880. Ueber anomale Dispersion im glühenden Natriumdampf. Ann. Phys. 246: $321-325$.

Ladenburg, Rudolf. 1908. Über die Absorption des Lichtes. Verh. Dtsch. Phys. Ges. 10: $550-561$.

Ladenburg, Rudolf. 1909a. Über Absorptionn und Magnetorotation in leuchtendem Wasserstoff. Phys. Z. 10: 497-499.

Ladenburg, Rudolf. 1909b. Die neueren Forschungen über die durch Licht- und Röntgenstrahlen hervorgerufenen Emission negativer Elektronen. Jahrbuch der Radioaktivität und Elektronik 6: 425-484.

Ladenburg, Rudolf. 1911. Astrophysikalische Bemerkungen im Anschluß an Versuche über Absorption und anomale Dispersion in leuchtendem Wasserstoff. Phys. Z. 12: 9-12.

Ladenburg, Albert. 1912. Lebenserinnerungen. Trewendt und Granier, Breslau.

Ladenburg, Rudolf. 1912. Über die anomale Dispersion und die magnetische Drehung der Polarisationsebene des leuchtenden Wasserstoffs, sowie die Verbreitung von Spektrallinien. Annalen der Physik 343: 249-318.

Ladenburg, Rudolf. 1914. Über die Zahl der an der Emission von Spektrallinien beteiligten Atome. Verh. Dtsch. Phys. Ges. 16: 765-779.

Ladenburg, Rudolf. 1920a. Bericht über die Bestimmung von Plancks elementarem Wirkungsquantum h. Jahrbuch der Radioaktivit $\bar{S} t$ und Elektronik 17: 93-145.

Ladenburg, Rudolf. 1920b. Die Elektronenordnung in den Elementen der großen Perioden. Die Naturwissenschaften 8: 5-11.

Ladenburg, Rudolf. 1921. Die quantentheoretische Deutung der Zahl der Dispersionselektronen. Z. Phys. 4: 451-468.

Ladenburg, Rudolf and Stanislau Loria. 1908a. Über die Dispersion des leuchtenden Wasserstoffs. Phys. Z. 9: 875-878.

Ladenburg, Rudolf and Stanislau Loria. 1908b. Über die Dispersion des leuchtenden Wasserstoffs. Verh. Dtsch. Phys. Ges. 10: 858-866.

Ladenburg, Rudolf and Rudolph Minkowski. 1921. Die Verdampfungswärme des Natriums und die Übergangswahrscheinlichkeit des Na-Atoms aus dem Resonanz- in der Normalzustand auf Grund optischer Messungen. Z. Phys. 6: 153-164.

Ladenburg, Rudolf and Fritz Reiche. 1913. Über selektive Absorption. Ann. Phys. 347: $181-209$.

Ladenburg, Rudolf and Fritz Reiche. 1923. Absorption, Zerstreuung und Dispersion in der Bohrschen Atomtheorie. Die Naturwissenschaften 11: 584-598.

Leeuwen, Hendrika van. 1916. Die Instabilität des Bohr-Debyeschen Wasserstoff-Moleküls und die Dispersion von Wasserstoff. Phys. Z. 17: 196-198. 
Loria, Stanislau. 1909. Quantitative Bestimmung der anomalen Dispersion des Lichtes in ges $\bar{S}$ ttigtem nicht leuchtendem Natriudampfe von der Temperatur $385 \circ$ C. Die Naturwissenschaften 11: 584-598.

Lommel, Eugen von. 1878a. Theorie der Absorption und Fluoreszenz. Ann. Phys. 239: $251-283$.

Lommel, Eugen von. 1878b. Theorie der normalen und anomalen Dispersion. Ann. Phys. 239: 339-356.

Lorentz, Hendrik A. 1892. La théorie électromagnétique de Maxwell et son application aux corps mouvants. Archives néerlandaises des sciences exactes et naturelles 25: 363-552.

Lorentz, Hendrik A. 1895. Versuch einer Theorie der elektrischen und optischen Erscheinungen in bewegten Körpern. E. J. Brill, Leiden.

Lummer, Otto R. and Ernst Pringsheim. 1903. Zur anomalen Dispersion der Gasen. Phys. Z. 4: $430-434$.

Lummer, Otto R. and Fritz Reiche. 1910. Über die Abhängigkeit der Strahlung einer Bunsenplatte (Bec-Meker) vom Ausstrahlungswinkel. Ann. Phys. 338: 857-870.

MacKinnon, Edward. 1977. Heisenberg, models and the rise of matrix mechanics. Historical Studies in the Physical Sciences 8: 135-188.

Mehra, Jagdish and Helmunt Rechenberg. 1982. The historical development of quantum theory. Volume 1. Part 1. The quantum theory of Planck, Einstein, Bohr, and Sommerfeld: its foundations and the rise of its difficulties 1900-1925. Springer, New York.

Natanson, Ladislas. 1907. On the electromagnetic theory of dispersion and extinction in gaseous bodies. Bulletin International de l'Academie des Scienes de Cracovie, Classe des Sciences Mathematiques et Naturelles: 316-326.

Nisio, Sigeko 1973. The formation of the Sommerfeld theory of 1916. Japanese Studies in the History of Science 12: 39-78.

Oseen, Carl W. 1915. Das Bohrsche Atommodell und die Maxwellschen Gleichungen. Phys. Z. 16: 395-405.

Planck, Max. 1906. Vorlesungen über die Theorie der Wärmestrahlung. Johann Ambrosius Barth, Leipzig.

Pflüger, Alexander. 1907. Über Absorption in lumineszierenden Gasen. Ann. Phys. 329: $515-526$.

Polvani, Giovanni. 1952. Mots de commémoration prononcés a la nouvelle de la mort du Prof. Luigi Puccianti. Il nuovo Cimento 9: 478-479.

Puccianti, Luigi. 1901. Dispersion anomala della ossiemoglobina. Il nuovo Cimento 1: 257264.

Puccianti, Luigi. 1905. Alcune osservazioni critiche ed esperienze nuove relative ai fondamenti della spettroscopia celeste. Il nuovo Cimento 9: 393-475.

Reiche, Fritz. 1921. Die Quantentheorie. Ihr Ursprung und die Entwicklung. Springer, Berlin.

Roschdestwensky, Dimitry. 1912. Anomale Dispersion in Natriumdampf. Ann. Phys. 344: 307-345.

Rosenfeld, Leon and Ulrich Hoyer (eds.). 1981. Work on Atomic Physics (1912-1917). In Niels Bohr Collected Works, edited by Finn Aaserud, vol. 2. North-Holland, Amsterdam.

Rubinowicz, Adalbert. 1917. Die Eingenschwingungen des Bohr-Debyeschen Wasserstoffmoleküls bei Berücksichtigung der Bewegung der Kerne. Phys. Z. 18: 187-195.

Sauter, Fritz. 1968. Arnold Sommerfeld. Gesammelte Schriften. 4 vols.. Vieweg und Sohn, Braunschweig.

Schaefer, Clemens. 1909a Dispersionstheorie und Serienspektren. Ann. Phys. 333: 421-435.

Schaefer, Clemens. 1909b. Zur Dispersionstheorie der Serienspektren. Ann. Phys. 334: 715722 .

Schaefer, Clemens. 1910. Über die Bestimmung der Elektronenzahl aus der Dispersion. Ann. Phys. 337: 883-888.

Schott, George A. 1908. Zur Erklärung der Spektrallinien. Phys. Z. 9: 214-216.

Seabrook, William B. 1941. Doctor Wood. Modern wizard of the laboratory. Charles Scribner's Sons, New York. 
Sellmeier, Wilhelm. 1872a. Über die durch die Aetherschwingungenerregten Mitschwingungen der Körpertheilchen und deren Rückwirkung auf die ersten, besonders zur Erklärung der Dispersion und ihrer Anomalien, I. Theil. Ann. Phys. 221: 399-421.

Sellmeier, Wilhelm. 1872b. Über die durch die Aetherschwingungenerregten Mitschwingungen der Körpertheilchen und deren Rückwirkung auf die ersten, besonders zur Erklärung der Dispersion und ihrer Anomalien, Fortsetzung. Ann. Phys. 221: 520-549.

Sellmeier, Wilhelm. 1872c. Über die durch die Aetherschwingungenerregten Mitschwingungen der Körpertheilchen und deren Rückwirkung auf die ersten, besonders zur Erklärung der Dispersion und ihrer Anomalien, II. Theil. Ann. Phys. 223: 386-403.

Sellmeier, Wilhelm. 1872d. Über die durch die Aetherschwingungenerregten Mitschwingungen der Körpertheilchen und deren Rückwirkung auf die ersten, besonders zur Erklärung der Dispersion und ihrer Anomalien, Schluss. Ann. Phys. 223: 525-554.

Sellmeier, Wilhelm. 1890. Die Sonne unter der Herrschaft der drei Planeten Venus, Erde und Jupiter. Schmidt, Halle.

Seth, Suman. 2010. Crafting the quantum. Arnold Sommerfeld and the practice of theory, 1 890-1926. The MIT Press, Cambridge, MA.

Shenstone, Allen G. 1973. Rudolf Ladenburg. In Complete dictionary of scientific biography, ed. Charles Gillispie. Charles Scribner's Sons, New York.

Sommerfeld, Arnold. 1915. Die allgemeine Dispersionsformel nach dem Bohrschen Modell. In Festschrift Julius Elster und Hans Geitel ed. K. Bergwitz: 549-584.

Sommerfeld, Arnold. 1916a. Zur Quantentheorie der Spektrallinien. Ann. Phys. 356: 1-94.

Sommerfeld, Arnold. 1916b. Zur Quantentheorie der Spektrallinien. Ann. Phys. 356: 125167.

Sommerfeld, Arnold. 1917. Die Drudesche Dispersionstheorie vom Standpunkte des Bohrschen Modelles und die Konstitution von $\mathrm{H}_{2}, \mathrm{O}_{2}$ und $\mathrm{N}_{2}$. Ann. Phys. 358: 497-550.

Sommerfeld, Arnold. 1919. Atombau und Spektrallinien. Vieweg und Sohn, Braunschweig.

Stark, Johannes. 1916. Beobachtungen über den zeitlichen Verlauf der Lichtemission in Spektralserien. Ann. Phys. 354: 731-768.

Stern, Otto and Max Volmer. 1919. Über die Abklingungszeit der Fluoreszenz. Phys. Z. 20: $183-188$.

Voigt, Woldemar. 1908. Magneto- und Elektro-Optik. Teubner, Leipzig.

Voigt, Woldemar. 1913. Die anormalen Zeemaneffekte der Spektrallinien von D-Typus. Ann. Phys. 347: 210-230.

Waldeck, Florian. 1920. Alte Mannheimer Familien. Max Hahn and Co., Mannheim.

Whittaker, Edmund. 1910. A history of the theories of aether and electricity: from the age of Descartes to the close of the nineteenth century. Longman, Gree and Co., London.

Wien, Wilhelm. 1919. Über Messungen der Leuchtdauer der Atome und der Dämpfung der Spektrallinie. II. Ann. Phys. 371: 597-637.

Winkelmann, Adolph. 1887. Notiz zur anomalen Dispersion glühender Metalldämpfe. Ann. Phys. 268: 439-442.

Wood, Robert W. 1901. On the production of a line spectrum by anomalous dispersion, and its application to the 'flash spectrum'. Philos. Mag. Series 6 1: 551-555.

Wood, Robert W. 1902. The anomalous dispersion of sodium vapour. Proc. R. .Soc. Lond. 69: $157-171$.

Wood, Robert W. 1904. A quantitative determination of the anomalous dispersion of sodium vapour in the visible and ultra-violet regions. Philos. Mag. Series 6 8: 293-324. 